\title{
The Cosmic Microwave Background
}

\author{
Ruth Durrer* \\ Université de Genève \\ Département de Physique Théorique \\ 24, Quai E. Ansrmet, 1211 Genève 4, Switzerland E-mail: \\ ruth.durrer@physics.unige.ch
}

\begin{abstract}
In these lectures on the cosmic microwave background (CMB) we shall first give an overview of cosmological perturbation theory. Then we derive CMB anisotropies in the instant decoupling approximation where we can obtain them by integrating lightlike geodesics from the last scattering surface until today. In a second step we use the Boltzmann equation to investigate also polarization and Silk damping. In the final lecture we discuss experimental data and parameter estimation from observations of CMB anisotropies and polarization.
\end{abstract}

Cargèse Summer School: Cosmology and Particle Physics Beyond the Standard Models June 30-August 112007

Cargèse, France

${ }^{*}$ Speaker. 


\section{Introduction}

The cosmic microwave background (CMB) has been discovered accidentally in 1965 by Penzias and Wilson [1] who obtained the Nobel prize 1978 for this discovery. It was the decisive evidence which convinced most physicists in favor of the Big Bang model, initiated by Friedmann and Lemaître and worked out be Gamov, Dicke, Peebles and others, as compared to a steady state cosmology which had been advocated by Fred Hoyle and collaborators.

More or less immediately after the discovery of the CMB cosmologists began to search for its fluctuations which must be there if the observed cosmic structures, galaxies, clusters, voids etc. have formed by gravitational instability from small initial perturbations. For a long time they found nothing apart from a dipole anisotropy on the level of $1.2 \times 10^{-3}$ which can be interpreted by the Doppler shift due to the motion of the earth with respect to the rest frame of the surface of last scattering. Finally, in 1992 the fluctuations have been found with the help of the COBE (Cosmic Background Explorer) Satellite mission from NASA [2]. During the last 15 years, the cosmic microwave background (CMB) has become the most important observational tool in cosmology. This is probably partly why G. Smoot has been attributed the Nobel prize of 2006 for this important discovery.

In these lectures, you will learn why CMB anisotropies and polarization are so important for cosmology and what we can learn from them. We shall assume that you are already familiar with Friedmann-Lemaitre (FL) cosmology and have some knowledge of inflation, since this has been treated in the course by Cliff Burgess. These lectures are based on my book [3] which contains of course much more than what is presented here. Especially, we shall not talk about CMB lensing and the CMB frequency spectrum. Furthermore, in this course we shall not derive the Boltzmann equation in any detail and we will not enter in Markov chain Monte Carlo methods.

However, We shall give a thorough overview of cosmological perturbation theory, the calculation of CMB anisotropies in the instantaneous recombination approximation and the CMB spectrum. We shall also discuss the Boltzmann equation which is needed to investigate polarization and Silk damping. Furthermore, we present the observational results and the principles of parameter estimation and degeneracies.

Notation: We denote spacetime indices by Greek letters and purely spatial indices by Latin letters. Spatial 3-vectors are written in bold. We choose the background metric

$$
d s^{2}=a^{2}(t)\left[-d t^{2}+\gamma_{i j} d x^{i} d x^{j}\right]=g_{\mu v} d x^{\mu} d x^{v},
$$

where $\gamma$ is the metric of a 3-space with constant curvature $K$. Note that $t$ is conformal time. We denote cosmic time by $\tau, d \tau=a d t$. An over-dot denotes the derivative w.r.t conformal time $t$. Latin $3 \mathrm{~d}$ indices are raised and lowered with the 3-metric $\gamma$ while Greek spacetime indices are raised and lowered with $g_{\mu \nu}$ respectively $g^{\mu \nu}$.

\section{Cosmological perturbation theory}

Fluctuations in the CMB are small. Therefore they can, to a good accuracy, be treated within first order perturbation theory. This leads to linear evolution equations for perturbation variables which can be solved to high accuracy with moderate numerical investment. This is one of the main 
reasons why observations of $\mathrm{CMB}$ fluctuations are so valuable. Comparing them with calculations we can accurately determine the values of the parameters of the underlying cosmological model and the initial perturbations. Therefore, linear cosmological perturbation theory is the basic tool to investigate CMB anisotropies. This section is devoted to a discussion of the main elements of cosmological perturbation theory.

\subsection{Definition of cosmological perturbations, gauges}

Denoting the cosmic scale factor by $a(t)$ and the metric of a 3 dimensional space of constant curvature by $\gamma_{i j}$ the most general metric of a perturbed Friedmann universe is of the form

$d s^{2}=a^{2}(t)\left[-(1+2 \Psi) d t^{2}+2 \sigma_{i} d t d x^{i}+(1-2 \Phi) \gamma_{i j} d x^{i} d x^{j}+2 H_{i j} d x^{i} d x^{j}\right]=\left(\bar{g}_{\mu v}+h_{\mu v}\right) d x^{\mu} d x^{v}$.

$H_{i j}$ is traceless, $H_{i}^{i}=H_{i j} \gamma^{i j}=0$.

The split into a background $\bar{g}_{\mu \nu}$ and a perturbation $h_{\mu \nu}$ is not unique. The only measurable geometry is the one described by the full metric $g_{\mu \nu}=\bar{g}_{\mu \nu}+h_{\mu \nu}$. The same is also true for the energy momentum tensor. The full physical fields $g_{\mu \nu}$ and $T_{\mu \nu}$ are related via Einstein's equation,

$$
G_{\mu v}=8 \pi G T_{\mu v}
$$

We can define a background metric and background energy momentum tensor by choosing a foliation of spacetime and averaging the metric and the energy momentum tensor over spatial slices, $g_{\mu \nu} \rightarrow \bar{g}_{\mu \nu}$ and $T_{\mu \nu} \rightarrow \bar{T}_{\mu \nu}$. In general, however, due to the non-linearity of Einstein's equation, the averaged metric and energy momentum tensor will not satisfy them,

$$
G_{\mu v}\left(\bar{g}_{\mu v}\right) \neq 8 \pi G \bar{T}_{\mu v}
$$

Let us call an averaging procedure 'admissible' if it obeys the following two conditions:

1. The averaged fields $\bar{g}$ and $\bar{T}$ satisfy Einstein's equation (which in this case reduce to the Friedmann equations).

2. The deviations of the averaged fields and the physical fields are small in the sens that

$$
\frac{\left|T_{\mu v}-\bar{T}_{\mu v}\right|}{\max _{\{\alpha \beta\}}\left\{\left|\bar{T}_{\alpha \beta}\right|\right\}} \sim \varepsilon \ll 1, \quad \text { and } \quad \frac{\left|g_{\mu v}-\bar{g}_{\mu v}\right|}{\max _{\{\alpha \beta\}}\left\{\bar{g}_{\alpha \beta}\right\}} \sim \varepsilon \ll 1 .
$$

There may be many different admissible averaging procedures (e.g., over different hyper-surfaces), leading to slightly different Friedmann-Lemaitre (FL) backgrounds. But since $|g-\bar{g}| / a^{2}$ is small, of order $\varepsilon$, the difference of the two FL backgrounds must also be small of order $\varepsilon$ and we can interpret it as part of the perturbation. We consider now a fixed admissible FL background $(\bar{g}, \bar{T})$ as chosen. Since the theory is invariant under diffeomorphisms (coordinate transformations), the perturbations are not unique. For an arbitrary diffeomorphism $\phi$ and its push forward $\phi_{*}$, the two metrics $g$ and $\phi_{*}(g)$ describe the same geometry. Since we have chosen the background metric $\bar{g}$, we only allow diffeomorphisms which leave $\bar{g}$ invariant i.e., which deviate only in first order from the identity. Such an 'infinitesimal' diffeomorphism can be represented as the infinitesimal flow of a vector field $X, \phi=\phi_{\varepsilon}^{X}$. Remember the definition of the flow: For the integral curve, $\gamma_{x}(s)$, of $X$ 
with starting point $p$, i.e., $\gamma_{x}(s=0)=p$ we have $\phi_{s}^{X}(p)=\gamma_{x}(s)$. In terms of the vector field $X$, to first order in $\varepsilon$, its push forward is then given by

$$
\phi_{*}=i d+\varepsilon L_{X}+\mathscr{O}\left(\varepsilon^{2}\right),
$$

where $L_{X}$ denotes the Lie derivative in direction $X$. The transformation $g \rightarrow \phi_{*}(g)$ is equivalent to $\bar{g}+\varepsilon a^{2} h \rightarrow \bar{g}+\varepsilon\left(a^{2} h+L_{X} \bar{g}\right)+\mathscr{O}\left(\varepsilon^{2}\right)$. Under an 'infinitesimal coordinate transformation' the metric perturbation $h$ therefore transforms as

$$
h \rightarrow h+a^{-2} L_{X} \bar{g} .
$$

In the context of cosmological perturbation theory, infinitesimal coordinate transformations are called 'gauge transformations'. The perturbation of an arbitrary tensor field $S=\bar{S}+\varepsilon S^{(1)}$ obeys the gauge transformation law

$$
S^{(1)} \rightarrow S^{(1)}+L_{X} \bar{S}
$$

Since every vector field $X$ generates a gauge transformation $\phi=\phi_{\varepsilon}^{X}$, we conclude that only perturbations of tensor fields with $L_{X} \bar{S}=0$ for all vector fields $X$, i.e., , with vanishing (or constant) 'background contribution' are gauge invariant. This result is called the 'Stewart-Walker Lemma' [4].

The gauge dependence of perturbations has caused many controversies in the literature, since it is often difficult to extract the physical meaning of gauge dependent perturbations, especially on super-horizon scales. This problem is solved by gauge invariant perturbation theory which we are going to use here. The advantage of the gauge-invariant formalism is that gauge-invariant variables have simple geometric and physical meanings and are not plagued by gauge modes. Although the derivation requires somewhat more work, the final system of perturbation equations is usually simple and well suited for numerical treatment. We shall also see, that on sub-horizon scales, the gauge-invariant matter perturbation variables approach the usual, gauge dependent ones. Since one of the gauge-invariant geometrical perturbation variables corresponds to the Newtonian potential, the Newtonian limit can be performed easily. (In contrast to synchronous gauge where the Newtonian potential is gauged to zero.)

First we note that all relativistic equations are covariant and can therefore be written in the form $S=0$ for some tensor field $S$. The corresponding background equation is $\bar{S}=0$, hence $S^{(1)}$ is gauge-invariant. It is thus always possible to express the perturbation equations in terms of gauge-invariant variables.

The principal sources of this section are the Refs. $[3,5,6,7,8]$ on gauge-invariant cosmological perturbation theory.

\subsection{Harmonic decomposition}

Since the $\{t=$ const. $\}$ hypersurfaces are homogeneous and isotropic, it is reasonable to perform a harmonic analysis for the perturbation variables defined on them. This means that we decompose a (spatial) tensor field on these hypersurfaces into components which transform irreducibly under translations and rotations. For functions (tensor fields of rank zero), in the case of vanishing spatial curvature, $K=0$, this is simply the Fourier decomposition,

$$
f(\mathbf{x}, t)=\frac{1}{(2 \pi)^{3}} \int d^{3} k f(\mathbf{k}, t) e^{-i \mathbf{k} \mathbf{x}} \text {. }
$$


If $K \neq 0$ it is the decomposition into eigenfunctions of the Laplacian,

$$
\Delta Q_{\mathbf{k}}^{(S)}=-k^{2} Q_{\mathbf{k}}^{(S)} .
$$

For $K=1$ the values $k$ are the discrete eigenvalues of the Laplacian on the 3 -sphere, $k^{2}=\ell(\ell+2)$ and for $K=-1$, they are bounded from below, $k^{2}>1$.

In addition, a tensorial variable (at fixed position $\mathbf{x}$ ) can be decomposed into irreducible components under the rotation group $S O(3)$.

For a spatial vector field, this is its decomposition into a gradient and a curl component,

$$
V_{i}=\nabla_{i} \varphi+B_{i}, \quad \text { where } \quad B_{\mid i}^{i}=0
$$

We use $X_{\mid i}$ to denote the three-dimensional covariant derivative of $X$. Here $\varphi$ is the spin 0 and $\mathbf{B}$ is the spin 1 component of the vector field $\mathbf{V}$.

For a spatial symmetric tensor field we have

$$
H_{i j}=H_{L} \gamma_{i j}+\left(\nabla_{i} \nabla_{j}-\frac{1}{3} \Delta \gamma_{i j}\right) H_{T}+\frac{1}{2}\left(H_{i \mid j}^{(V)}+H_{j \mid i}^{(V)}\right)+H_{i j}^{(T)}
$$

where

$$
H_{i}^{(V) \mid i}=H_{i}^{(T)^{i}}=H_{i \mid j}^{(T)^{j}}=0
$$

Here $H_{L}$ and $H_{T}$ are spin 0 components, $H_{i}^{(V)}$ is the spin 1 component and $H_{i j}^{(T)}$ is the spin 2 component of the tensor field $H$.

We shall not need higher tensors (or spinors). As a basis for vector and tensor modes we use the vector and tensor type eigenfunctions of the Laplacian,

$$
\Delta Q_{j}^{(V)}=-k^{2} Q_{j}^{(V)} \quad \text { and } \quad \Delta Q_{j i}^{(T)}=-k^{2} Q_{j i}^{(T)}
$$

where $Q_{j}^{(V)}$ is a transverse vector, $Q_{j}^{(V) \mid j}=0$ and $Q_{j i}^{(T)}$ is a symmetric transverse traceless tensor, $Q_{j}^{(T) j}=Q_{j i}^{(T) \mid i}=0$. Both, $Q_{j}^{(V)}$ and $Q_{j i}^{(T)}$ have two degrees of freedom. In the case of vanishing curvature we can use an orthonormal basis $\mathbf{e}^{(1)}, \mathbf{e}^{(2)}$ in the plane normal to $\mathbf{k}$ and we can define helicity basis vectors,

$$
\mathbf{e}^{ \pm}=\frac{1}{\sqrt{2}}\left(\mathbf{e}^{(1)} \pm i \mathbf{e}^{(2)}\right)
$$

In curved spaces the definition of the helicity basis is analogous, but somewhat more involved. Since we shall not need the explicite form of this basis, we shall not enter into this. Vector perturbations can be expanded in terms of this basis, while tensor perturbations are expanded either in terms of the standard tensor basis given by

$$
e_{i j}^{d}=\frac{1}{2}\left[\mathbf{e}_{i}^{(1)} \mathbf{e}_{j}^{(1)}-\mathbf{e}_{i}^{(2)} \mathbf{e}_{j}^{(2)}\right], \quad e_{i j}^{\times}=\frac{1}{2}\left[\mathbf{e}_{i}^{(1)} \mathbf{e}_{j}^{(2)}+\mathbf{e}_{i}^{(2)} \mathbf{e}_{j}^{(1)}\right],
$$

or also in terms of a helicity basis defined by

$$
e_{i j}^{(+2)}=\mathbf{e}_{i}^{+} \mathbf{e}_{j}^{+}=e_{i j}^{d}+i e_{i j}^{\times}, \quad e_{i j}^{(-2)}=\mathbf{e}_{i}^{-} \mathbf{e}_{j}^{-}=e_{i j}^{d}-i e_{i j}^{\times} .
$$


We can develop the vector and tensor basis functions as

$$
\begin{aligned}
& Q_{j}^{(V)}=Q^{(1)} \mathbf{e}_{j}^{(1)}+Q^{(2)} \mathbf{e}_{j}^{(2)}=Q^{(+)} \mathbf{e}_{j}^{(+)}+Q^{(-)} \mathbf{e}_{j}^{(-)} \\
& Q_{j i}^{(T)}=Q^{(d)} e_{i j}^{(d)}+Q^{(\times)} e_{i j}^{(\times)}=Q^{(+2)} e_{i j}^{(+2)}+Q^{(-2)} e_{i j}^{(-2)} .
\end{aligned}
$$

The components in the 'helicity basis', $\mathbf{e}^{( \pm)}$and $e_{i j}^{( \pm 2)}$ simply transform with a phase $e^{ \pm i \varphi}$, and $e^{ \pm 2 i \varphi}$ respectively under rotations around $\mathbf{k}$ with angle $\varphi$. Hence vector perturbations are spin 1 fields while tensor perturbations are spin 2 fields. The functions $Q^{(+)}$and $Q^{(+2)}$ have spin up, $m=+1$ and $m=+2$ respectively, while $Q^{(-)}$and $Q^{(-2)}$ have spin down. Scalar perturbations of course have spin zero.

As in Eqs. (2.8) and (2.9), we can construct scalar-type vectors and traceless symmetric tensors and vector-type traceless symmetric tensors. To this goal we define

$$
\begin{aligned}
& Q_{j}^{(S)} \equiv-k^{-1} Q_{\mid j}^{(S)}, \quad Q_{i j}^{(S)} \equiv k^{-2} Q_{\mid i j}^{(S)}+\frac{1}{3} \gamma_{i j} Q^{(S)} \text { and } \\
& Q_{i j}^{(V)} \equiv-\frac{1}{2 k}\left(Q_{i \mid j}^{(V)}+Q_{j \mid i}^{(V)}\right) .
\end{aligned}
$$

In the following we shall extensively use this decomposition and write down the perturbation equations for a given mode $k$.

The decomposition of the $k$-mode of a vector field is then of the form

$$
V_{i}=V Q_{i}^{(S)}+V^{(V)} Q_{i}^{(V)} .
$$

The decomposition of a tensor field is given by (compare Eq. (2.9))

$$
H_{i j}=H_{L} Q^{(S)} \gamma_{i j}+H_{T} Q_{i j}^{(S)}+H^{(V)} Q_{i j}^{(V)}+H^{(T)} Q_{i j}^{(T)} .
$$

Here $B, B^{(V)}, H_{L}, H_{T}, H^{(V)}$ and $H^{(T)}$ are functions of $t$ and $\mathbf{k}$.

This decomposition is very useful, since scalar, vector and tensor amplitudes of each mode $\mathbf{k}$ evolve independently, obeying ordinary differential equations in time.

\subsection{Metric perturbations}

Perturbations of the metric are of the form

$$
g_{\mu v}=\bar{g}_{\mu v}+a^{2} h_{\mu v} .
$$

We parameterize them as

$$
h_{\mu \nu} d x^{\mu} d x^{\nu}=-2 A d t^{2}-2 B_{i} d t d x^{i}+2 H_{i j} d x^{i} d x^{j},
$$

and we decompose the perturbation variables $B_{i}$ and $H_{i j}$ according to (2.19) and (2.20).

Let us now investigate the behavior of $h_{\mu v}$ under gauge transformations. We set the vector field defining the gauge transformation to

$$
X=T \partial_{t}+L^{i} \partial_{i}
$$


Using the definition of the Lie derivative, we obtain

$$
\begin{aligned}
L_{\mathbf{X}} \bar{g}= & a^{2}\left[-2(\mathscr{H} T+\dot{T}) d t^{2}+2\left(\dot{L}_{i}-T_{, i}\right) d t d x^{i}\right. \\
& \left.+\left(2 \mathscr{H} T \gamma_{i j}+L_{i \mid j}+L_{j \mid i}\right) d x^{i} d x^{j}\right] .
\end{aligned}
$$

Comparing this with (2.22) and using (2.5), we obtain

$$
\begin{aligned}
A & \rightarrow A+\mathscr{H} T+\dot{T}, \\
B_{i} & \rightarrow B_{i}-\dot{L}_{i}+T,{ }_{i}, \\
H_{i j} & \rightarrow H_{i j}+\frac{1}{2}\left(L_{i \mid j}+L_{j \mid i}\right)+\mathscr{H} T \gamma_{i j} .
\end{aligned}
$$

Using the decompositions (2.19) and (2.20) for $B_{i}$ and $H_{i j}$, this implies the following behavior of the perturbation variables under gauge transformations (we also decompose the vector $L_{i}=$ $\left.L Q_{i}^{(S)}+L^{(V)} Q_{i}^{(V)}\right)$ :

$$
\begin{aligned}
A & \rightarrow A+\mathscr{H} T+\dot{T}, \\
B & \rightarrow B-\dot{L}-k T, \quad B^{(V)} \rightarrow B^{(V)}-\dot{L}^{(V)}, \\
H_{L} & \rightarrow H_{L}+\mathscr{H} T+\frac{k}{3} L, \quad H_{T} \rightarrow H_{T}-k L, \\
H^{(V)} & \rightarrow H^{(V)}-k L^{(V)} \quad H^{(T)} \rightarrow H^{(T)} .
\end{aligned}
$$

Two scalar and one vector variable can be set to zero by gauge transformations.

To fix the scalar perturbations of the metric, one often chooses the gauge transformation $k L=$ $H_{T}$ and $k T=B-\dot{L}$, so that the transformed variables $H_{T}$ and $B$ vanish. In this gauge (longitudinal gauge), scalar perturbations of the metric are of the form $\left(\left.H_{T}\right|_{\text {long }}=\left.B\right|_{\text {long }}=0\right)$ :

$$
h_{\mu \nu}^{(S)}=-2 \Psi d t^{2}-2 \Phi \gamma_{i j} d x^{i} d x^{j} .
$$

$\Psi$ and $\Phi$ are the so-called Bardeen potentials. In a generic gauge the Bardeen potentials are given by

$$
\begin{aligned}
& \Psi=A-\mathscr{H} k^{-1} \sigma-k^{-1} \dot{\sigma} \\
& \Phi=-H_{L}-\frac{1}{3} H_{T}+\mathscr{H} k^{-1} \sigma=-\mathscr{R}+\mathscr{H} k^{-1} \sigma,
\end{aligned}
$$

where $\sigma=k^{-1} \dot{H}_{T}-B$, is the scalar potential for the shear of the hypersurface of constant time. A short calculation shows that $\Psi$ and $\Phi$ are indeed invariant under gauge transformations (for more details see [3]).

In a FL universe the Weyl tensor (see e.g., [9]) vanishes. It therefore is a gauge-invariant perturbation. For scalar perturbations one finds

$$
E_{i j} \equiv C_{i v j}^{\mu} u_{\mu} u^{v}=-C_{i 0 j}^{0}=\frac{-1}{2}\left[(\Psi+\Phi)_{\mid i j}-\frac{1}{3} \Delta(\Psi+\Phi) \gamma_{i j}\right]
$$

All other components vanish.

For vector perturbations it is convenient to set $k L^{(V)}=H^{(V)}$ so that $H^{(V)}$ vanishes and we have

$$
h_{\mu \nu}^{(V)} d x^{\mu} d x^{\nu}=2 a^{2} \sigma^{(V)} Q_{i}^{(V)} d t d x^{i} .
$$


We shall call this gauge the "vector gauge".

The Weyl tensor from vector perturbation is given by

$$
\begin{aligned}
E_{i j} & =-C_{i 0 j}^{0}=\frac{-k}{2} \dot{\sigma}^{(V)} Q_{i j}^{(V)} \\
B_{i j} & \equiv \frac{1}{2} \varepsilon_{i v}{ }^{\rho \sigma} C_{\rho \sigma}{ }^{j \alpha} u_{v} u_{\alpha}=\varepsilon_{i l m} C_{j l m}^{0} \\
& =\frac{-k}{2} \sigma^{(V)} \varepsilon_{i l m}\left[Q_{j l \mid m}^{(V)}-Q_{j m \mid l}^{(V)}-\frac{1}{3} \gamma_{j l} Q_{m \mid k}^{(V) k}+\frac{1}{3} \gamma_{j m} Q_{l \mid k}^{(V) k}\right] .
\end{aligned}
$$

Note that from their definition $E_{i j}$ and $B_{i j}$ are symmetric and since $u=\left(u^{0}, \mathbf{0}\right)$ to lowest order, only $C_{i 0 j}^{0}$ and $C_{i l m}^{0}$ respectively contribute. The tensors $E_{i j}$ and $B_{i j}$, determine the Weyl tensor fully.

Clearly there are no tensorial (spin 2) gauge transformations and hence $H_{i j}^{(T)}$ is gauge-invariant. The expression for the Weyl tensor from tensor perturbation is

$$
\begin{aligned}
E_{i j} & =\frac{1}{2}\left(\partial_{t}^{2}-k^{2}\right) H^{(T)} Q_{i j}^{(T)}, \\
B_{i j} & =-\dot{H}^{(T)} \varepsilon_{i l m}\left[Q_{j l \mid m}^{(T)}-Q_{j m \mid l}^{(T)}\right] .
\end{aligned}
$$

\subsection{Perturbations of the energy momentum tensor}

Let $T_{v}^{\mu}=\bar{T}_{v}^{\mu}+\theta_{v}^{\mu}$ be the full energy momentum tensor. We define its energy density $\rho$ and its energy flux 4-vector $u$ as the time-like eigenvalue and eigenvector of $T_{v}^{\mu}$ :

$$
T_{v}^{\mu} u^{v}=-\rho u^{\mu}, \quad u^{2}=-1 .
$$

We then parameterize their perturbations by

$$
\rho=\bar{\rho}(1+\delta), \quad u=u^{0} \partial_{t}+u^{i} \partial_{i}
$$

To first order, the component $u^{0}$ is fixed by the normalization condition,

$$
u^{0}=\frac{1}{a}(1-A) .
$$

The spatial components provide new perturbations. We set

$$
u^{i}=\frac{1}{a} v^{i}=\frac{1}{a}\left(v Q^{(S) i}+v^{(V)} Q^{(V) i}\right) .
$$

$P_{v}^{\mu} \equiv u^{\mu} u_{v}+\delta_{v}^{\mu}$ is the projection tensor onto the subspace of tangent space normal to $u$. We define the stress tensor

$$
\tau^{\mu v}=P_{\alpha}^{\mu} P_{\beta}^{v} T^{\alpha \beta}
$$

With this we can write

$$
T_{v}^{\mu}=\rho u^{\mu} u_{v}+\tau_{v}^{\mu} .
$$

In the unperturbed case we have $\tau_{\mu}^{0}=\tau_{0}^{\mu}=0$ and $\tau_{j}^{i}=\bar{P} \delta_{j}^{i}$. Including first order perturbations, the components $\tau_{0 \mu}$ are determined by the perturbation variables which we have already introduced. We obtain

$$
\tau_{0}^{0}=0, \quad \text { and } \quad \tau_{0}^{j}=-\bar{P} v^{j}, \quad \tau_{j}^{0}=\bar{P}\left(v_{j}-B_{j}\right) .
$$


But $\tau_{j}^{i}$ contains in general new perturbations. We define

$$
\tau_{j}^{i}=\bar{P}\left[\left(1+\pi_{L}\right) \delta_{j}^{i}+\Pi_{j}^{i}\right], \quad \text { with } \quad \Pi_{i}^{i}=0 .
$$

From our definitions we can determine the perturbations of the energy momentum tensor. A short calculation gives

$$
\begin{aligned}
T_{0}^{0} & =-\bar{\rho}(1+\delta), \quad T_{j}^{0}=(\bar{\rho}+\bar{P})\left(v_{j}-B_{j}\right), \quad T_{0}^{j}=-(\bar{\rho}+\bar{P}) v^{j} \quad \text { and } \\
T_{j}^{i}{ }_{j} & =\bar{P}\left[\left(1+\pi_{L}\right) \delta_{j}^{i}+\Pi_{j}^{i}\right] .
\end{aligned}
$$

The traceless part of the stress tensor, $\Pi_{j}^{i}$, is called the anisotropic stress tensor. We decompose it as

$$
\Pi_{j}^{i}=\Pi Q_{j}^{(S) i}+\Pi^{(V)} Q_{j}^{(V) i}+\Pi^{(T)} Q_{j}^{(T) i} .
$$

We now study the gauge transformation properties of these perturbation variables. First we note that $\rho$ is a scalar, hence $L_{X} \bar{\rho}=\dot{\bar{\rho}} T=-3(1+w) \mathscr{H} \bar{\rho} T$. Here we made use of the background energy conservation equation, $\dot{\bar{\rho}}=-3(\bar{\rho}+\bar{P}) \mathscr{H}=-3 \bar{\rho}(1+w) \mathscr{H}$, with $w=\bar{P} / \bar{\rho}$. The same is true for $\bar{P}\left(1+\pi_{L}\right)$ which is $1 / 3$ of the trace of $\tau^{\mu}{ }_{v}$. With $c_{s}^{2} \equiv \dot{\bar{P}} / \dot{\bar{\rho}}$, we obtain $L_{X} \bar{P}=\dot{\bar{P}} T=$ $-3 \frac{c_{s}^{2}}{w}(1+w) \mathscr{H} \bar{P} T$. The background contribution to the anisotropic stress tensor, $\Pi_{v}^{\mu}=\tau_{v}^{\mu}-$ $\frac{1}{3} \tau_{\alpha}^{\alpha} \delta_{v}^{\mu}$, vanishes, hence the Stewart-Walker lemma implies that $\Pi_{v}^{\mu}$ is gauge-invariant. lemma. For perfect fluids $\Pi_{v}^{\mu}=0$. For the velocity we use $L_{X} \bar{u}=[X, \bar{u}]=\left(-T \dot{a} a^{-2}-a^{-1} \dot{T}\right) \partial_{t}-a^{-1} \dot{L}^{i} \partial_{i}$. Inserting our decomposition into scalar, vector and tensor perturbation variables for a fixed mode $\mathbf{k}$, we obtain finally the following transformation behavior

$$
\begin{aligned}
\delta & \rightarrow \delta-3(1+w) \mathscr{H} T, \quad \pi_{L} \rightarrow \pi_{L}-3 \frac{c_{s}^{2}}{w}(1+w) \mathscr{H} T, \\
v & \rightarrow v-\dot{L}, \quad \Pi \rightarrow \Pi \\
v^{(V)} & \rightarrow v^{(V)}-\dot{L}^{(V)}, \quad \Pi^{(V)} \rightarrow \Pi^{(V)}, \\
\Pi^{(T)} & \rightarrow \Pi^{(T)} .
\end{aligned}
$$

Apart from the anisotropic stress perturbations, there is only one gauge-invariant variable which can be obtained from the energy momentum tensor alone, namely

$$
\Gamma=\pi_{L}-\frac{c_{s}^{2}}{w} \delta
$$

One can show (see [3]) that $\Gamma$ is proportional to the divergence of the entropy flux of the perturbations. Adiabatic perturbations are characterized by $\Gamma=0$.

Gauge-invariant density and velocity perturbations can be found by combining $\delta, v$ and $v_{i}^{(V)}$ with metric perturbations. We shall use

$$
\begin{aligned}
V & \equiv v-\frac{1}{k} \dot{H}_{T}=v^{\text {long }}, \\
D_{s} & \equiv \delta+3(1+w) \mathscr{H}\left(k^{-2} \dot{H}_{T}-k^{-1} B\right) \equiv \delta^{\text {long }} \\
D & \equiv \delta^{\text {long }}+3(1+w) \frac{\mathscr{H}}{k} V=\delta+3(1+w) \frac{\mathscr{H}}{k}(v-B)
\end{aligned}
$$




$$
\begin{aligned}
& =D_{s}+3(1+w) \frac{\mathscr{H}}{k} V \\
D_{g} & \equiv \delta+3(1+w)\left(H_{L}+\frac{1}{3} H_{T}\right)=\delta^{\text {long }}-3(1+w) \Phi \\
& =D_{s}-3(1+w) \Phi \\
V^{(V)} & \equiv v^{(V)}-\frac{1}{k} \dot{H}^{(V)}=v^{(\mathrm{vec})} \\
\Omega & \equiv v^{(V)}-B^{(V)}=v^{(\mathrm{vec})}-B^{(V)} \\
\Omega-V^{(V)} & =\sigma^{(V)}
\end{aligned}
$$

Here $v^{\text {long }}, \delta^{\text {long }}$ and $v^{(\mathrm{vec})}$ are the velocity (and density) perturbations in longitudinal and vector gauge respectively, and $\sigma^{(V)}$ is the metric perturbation in vector gauge and the vector type contribution to the shear of the $t=$ constant hyper-surfaces.

These variables can be interpreted nicely in terms of gradients of the energy density and the shear and vorticity of the velocity field [10].

We now want to show that on scales much smaller than the Hubble scale, $k \gg \mathscr{H} \sim t^{-1}$, metric perturbations are much smaller than $\delta$ and $v$ and we can thus neglect the difference between different gauges and/or gauge-invariant variables. This is especially important when comparing experimental results with gauge-invariant calculations. Let us neglect spatial curvature in the following order of magnitude argument. Then, the perturbations of the Einstein tensor are a combination of second derivatives of the metric perturbations, $\mathscr{H}$ times first derivatives and $\mathscr{H}^{2}$ or $\mathscr{\mathscr { H }}$ times metric perturbations. The first order perturbation of Einstein's equations therefore generically yield the following order of magnitude estimate $8 \pi G \delta T_{\mu \nu}=\delta G_{\mu \nu}$ :

$$
\begin{aligned}
\mathscr{O}\left(\frac{\delta \rho}{\rho}\right) \underbrace{\mathscr{O}(8 \pi G \rho)}_{\mathscr{O}\left(a^{2} / t^{2}\right)} & =\mathscr{O}\left(\frac{1}{t^{2}} a^{2} h+\frac{k}{t} a^{2} h+k^{2} a^{2} h\right) \\
\mathscr{O}\left(\frac{\delta \rho}{\rho}\right) & =\mathscr{O}\left(h+k t h+(k t)^{2} h\right) .
\end{aligned}
$$

For $k t \gg 1$ this gives $\mathscr{O}(\delta, v)=\mathscr{O}\left(\frac{\delta T_{\mu v}}{\rho}\right)=\mathscr{O}\left((k t)^{2} h\right) \gg \mathscr{O}(h)$. Therefore, on sub-horizon scales the differences between $\delta, \delta^{\text {long }}, D_{g}$ and $D$ are negligible as well as the differences between $v$ and $V$ or $v^{(V)}, V^{(V)}$ and $\Omega^{(V)}$. Since measurements of density and velocity perturbations can only be made on sub-horizon scales, we may therefore use any of the gauge-invariant perturbation variables to compare with measurements.

\subsection{The perturbation equations}

We do not derive the first order perturbations of Einstein's equations. By elementary algebraic methods, this is quite lengthy and cumbersome. However, we recommend the student to simply determine $\delta G_{\mu \nu}$ in longitudinal (vector) gauge using some algebraic package like Maple or Mathematica and then write down the resulting Einstein equations using gauge-invariant variables. Since we know that these variables do not depend on the coordinates chosen, the equations obtained in this way are valid in any gauge. Here, we just present the resulting equations in gauge-invariant form. A rapid derivation by hand is possible using the $3+1$ formalism of general relativity and 
working with Cartan's formalism for the Riemann curvature, see [11]. In order to simplify the notation, we suppress the over-bar on background quantities whenever this does not lead to confusion.

\subsubsection{Einstein's equations}

The constraints: The Einstein equations $G_{0 \mu}=8 \pi G T_{0 \mu}$ lead to two scalar and one vector constraint equations,

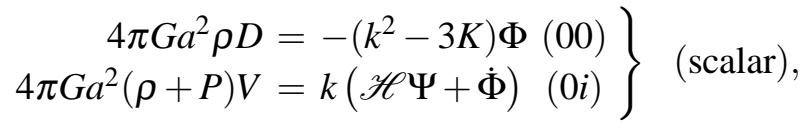

$$
\begin{aligned}
& 8 \pi G a^{2}(\rho+P) \Omega=\frac{1}{2}\left(2 K-k^{2}\right) \sigma^{(V)} \quad(0 i) \quad \text { (vector). }
\end{aligned}
$$

The dynamical equations: The Einstein equations $G_{i j}=8 \pi G T_{i j}$ provide two scalar, one vector and one tensor perturbation equation,

scalar:

$$
\begin{aligned}
& k^{2}(\Phi-\Psi)= 8 \pi G a^{2} P \Pi^{(S)}, \\
& \ddot{\Phi}+2 \mathscr{H} \dot{\Phi}+\mathscr{H} \dot{\Psi}+\left[2 \mathscr{\mathscr { C }}+\mathscr{H}^{2}-\frac{k^{2}}{3}\right] \Psi= \\
& 4 \pi G a^{2} \rho\left[\frac{1}{3} D+c_{s}^{2} D_{s}+w \Gamma\right],
\end{aligned}
$$

vector:

$$
k\left(\dot{\sigma}^{(V)}+2 \mathscr{H} \sigma^{(V)}\right)=8 \pi G a^{2} P \Pi^{(V)},
$$

tensor:

$$
\ddot{H}^{(T)}+2 \mathscr{H} \dot{H}^{(T)}+\left(2 K+k^{2}\right) H^{(T)}=8 \pi G a^{2} P \Pi^{(T)},
$$

The second dynamical scalar equation is somewhat cumbersome and not often used, since we may use one of the conservation equations given below instead. For the derivation of the perturbed Einstein equation the following relations are useful. They can be derived from the Friedmann equations.

$$
\begin{aligned}
4 \pi G a^{2} \rho(1+w) & =\mathscr{H}^{2}-\dot{\mathscr{H}}+K, \\
\dot{\mathscr{H}} & =-\frac{1+3 w}{2}\left(\mathscr{H}^{2}+K\right), \\
4 \pi G a^{2} \rho(1+w) 3 c_{s}^{2} & =\frac{\ddot{\mathscr{H}}}{\mathscr{H}}-\dot{\mathscr{H}}-\mathscr{H}^{2}-K, \\
c_{s}^{2} & =\frac{\frac{\mathscr{\mathscr { H }}}{\mathscr{H}}-\dot{\mathscr{H}}-\mathscr{H}^{2}-K}{3\left[\mathscr{H}^{2}-\dot{\mathscr{H}}+K\right] .}
\end{aligned}
$$

For the calculations below we shall also make use of

$$
\dot{w}=3\left(w-c_{s}^{2}\right)(1+w) \mathscr{H} .
$$


Note that for perfect fluids, where $\Pi_{j}^{i} \equiv 0$, we have $\Phi=\Psi$. As we shall see below, for perfect fluids with $\Gamma=\Pi=0$, the behavior of scalar perturbations is given by $\Psi$ which describes a damped wave propagating with speed $c_{s}^{2}$.

Tensor perturbations are given by $H^{(T)}$ which for perfect fluids also obeys a damped wave equation propagating with the speed of light. On small scales (over short time periods) when $t^{-2} \lesssim 2 K+k^{2}$, the damping term can be neglected and $H_{i j}$ represents propagating gravitational waves. For vanishing curvature or $k^{2} \gg K$, small scales are just the sub-Hubble scales, $k t \gtrsim 1$. For $K<0$, waves oscillate with a somewhat smaller frequency, $\omega=\sqrt{2 K+k^{2}}<k$, while for $K>0$ the frequency is somewhat higher than $k$.

Vector perturbations of a perfect fluid are determined by the $\sigma^{(V)}$-equation, Eq. (2.67), which implies $\sigma^{(V)} \propto 1 / a^{2}$. Hence vector perturbations do not oscillate, they simply decay.

\subsubsection{Energy momentum conservation}

The conservation equations, $T_{; v}^{\mu v}=0$ (here $; v$ denotes the four-dimensional covariant derivative) lead to the following perturbation equations:

$$
\begin{array}{r}
\dot{D}_{g}+3\left(c_{s}^{2}-w\right) \mathscr{H} D_{g}+(1+w) k V+3 w \mathscr{H} \Gamma=0 \\
\dot{V}+\mathscr{H}\left(1-3 c_{s}^{2}\right) V=k\left(\Psi+3 c_{s}^{2} \Phi\right)+\frac{c_{s}^{2} k}{1+w} D_{g} \\
+\frac{w k}{1+w}\left[\Gamma-\frac{2}{3}\left(1-\frac{3 K}{k^{2}}\right) \Pi\right] \\
\dot{\Omega}+\left(1-3 c_{s}^{2}\right) \mathscr{H} \Omega=-\frac{w}{2(1+w)}\left(k-\frac{2 K}{k}\right) \Pi^{(V)}
\end{array} \quad \text { (vector) }
$$

It is sometimes also useful to express the scalar conservation equations in terms of the variable pair $(D, V)$. Using $D=D_{g}+3(1+w)\left[\mathscr{H} k^{-1} V+\Phi\right]$ in (2.74) one obtains after some algebra and making use of the $(0 i)$ constraint equation (2.63)

$$
\begin{aligned}
\dot{D}-3 w \mathscr{H} D & =-\left(1-\frac{3 K}{k^{2}}\right)[(1+w) k V+2 \mathscr{H} w \Pi], \\
\dot{V}+\mathscr{H} V & =k\left[\Psi+\frac{c_{s}^{2}}{1+w} D+\frac{w}{1+w} \Gamma-\frac{2}{3}\left(1-\frac{3 K}{k^{2}}\right) \frac{w}{1+w} \Pi\right] .
\end{aligned}
$$

The conservation equations can of course also be obtained from the Einstein equations since they are equivalent to the contracted Bianchi identities. $\Gamma$ and $\Pi$ have to be determined via matter equations. For example, for adiabatic perturbations of an ideal fluid one has $\Gamma=\Pi=0$.

One can now use the (00) and (0i) Einstein equations, (2.63) to derive a second order equation for $\Phi$ and $\Psi$ from the conservation equations. Eliminating finally $\Phi$ with the $i \neq j$ Einstein equation,(2.65) yields the Bardeen equation,

$$
\ddot{\Psi}+3\left(1+c_{s}^{2}\right) \mathscr{H} \dot{\Psi}+\left[3\left(c_{s}^{2}-w\right) \mathscr{H}^{2}-\left(2+3 w+3 c_{s}^{2}\right) K+c_{s}^{2} k^{2}\right] \Psi=\mathscr{S}(\Pi, \Gamma)
$$

The right hand side is a 'source term' linear in $\Gamma$ and $\Pi$. It is very complicated and not very illuminating, therefore we do not write it down here.

For scalar perturbations we have in principle 4 independent equations and 6 variables. For vector perturbations we have 2 equations and 3 variables, while for tensor perturbations we have 
1 equation and 2 variables. To close the system we must add matter equations. The simplest prescription is to set $\Gamma=\Pi_{i j}=0$. This matter equation, which describes adiabatic perturbations of a perfect fluid gives us exactly two additional equations for scalar perturbations and one each for vector and tensor perturbations. If $c_{s}^{2} \neq 0$ the scalar equation (2.78) is a wave equation like the tensor equation discussed above. The first order term, $3\left(1+c_{s}^{2}\right) \mathscr{H}$ describes damping due to expansion, and the term in square brackets is of the form $m^{2}(t)+c_{s}^{2} k^{2}$, where the contributions proportional to $K$ and $\mathscr{H}^{2}$ are interpreted as a time dependent 'mass term'. If $K=0$ and $c_{s}^{2}=w$ the mass term vanishes and the Bardeen potential simply performs damped acoustic oscillations. This scalar wave equation describes the 'acoustic oscillations' of the fluid where the fluid pressure counter acts gravitational collapse. The vector perturbation equation, however, is of first order. $\Pi^{(V)}=0$ implies $\sigma^{(V)} \propto 1 / a^{2}$ and $\Omega \propto a^{-1+3 c_{s}^{2}}$. Hence vector perturbations simply decay if there are no anisotropic stresses to source them.

Another simple example is a universe with matter content given by a scalar field which is relevant for inflation [3]. More complicated are several interacting particle species of which some have to be described by a Boltzmann equation. This is the actual universe at late times, $z \lesssim 10^{7}$.

\subsection{Simple but important examples}

\subsubsection{Free gravity waves}

If there are no anisotropic stresses, like for example for a perfect fluid or a scalar field, and if curvature can be neglected, $K=0$ and expansion can be approximated by a simple power law, $a(t) \propto t^{q}$, the tensor perturbation equation reduces to

$$
\ddot{H}^{(T)}+\frac{2 q}{t} \dot{H}^{(T)}+k^{2} H^{(T)}=0 .
$$

The general solution of this equation can be given in terms of spherical Bessel functions,

$$
H^{(T)}=\frac{k t}{a}\left[A j_{q-1}(k t)+B j_{-q}(k t)\right] .
$$

Here $j_{v}$ denotes the spherical Bessel function of index $v$, see e.g., [12]. The second term diverges if $q>1 / 2$, for example in a radiation dominated universe where $q=1$, or in a matter dominated universe where $q=2$. (Not, however, in an inflating universe where $q<0$.) Therefore, in a radiation (or matter) dominated universe we have to set $B=0$, so that $H^{(T)}(t) \rightarrow$ const. for $t \rightarrow 0$. At late time we then find

$$
H^{(T)} \simeq \frac{A}{a} \sin \left(k t-\frac{q-1}{2} \pi\right), \quad k t \gg 1 .
$$

The energy density in gravitational waves per logarithmic frequency interval is then given by

$$
\frac{d \rho_{H}}{d \log k}=2 \frac{4 \pi\left\langle\left|\frac{d}{d \tau} H^{(T)}\right|^{2}\right\rangle k^{3}}{16 \pi G}=\frac{A^{2}(k) k^{5}}{4 G a^{4}} .
$$

Here $\frac{d}{d \tau}=a^{-1} \frac{d}{d t}$ is the derivative with respect to cosmic time and the factor $4 \pi k^{3}$ is the phase space volume per logarithmic frequency interval. The factor 2 comes from the two helicity modes. The brackets, $\langle\cdots\rangle$ indicate an average over several periods of the wave as well as an ensemble average (see Section 3.2). 
Note that $\rho_{H} \propto a^{-4}$ as we expect it for a component of massless particles. Furthermore, if the gravity wave power spectrum is scale invariant on large scales, $\left\langle\left|H^{(T)}\right|^{2}\right\rangle k^{3} \propto \frac{1}{2} A^{2}(k) k^{3+2 q}\left(t^{q} / a\right)^{2}=$ const. for $k t \ll 1$ and $n_{T}=0$ (see Section 3.2). This implies that $A^{2}(k) \propto k^{-3-2 q}$. Hence on scales which enter the horizon in the matter dominated era, $q=2$, the energy density decays with the wavenumber like $k^{-2}$ while it is independent of scale for wave numbers which enter the horizon in the radiation dominated era, $q=1$. A rough estimate neglecting the matter/radiation transition gives for scales which enter the horizon in the radiation dominated era, $k>\mathscr{H}_{\mathrm{eq}} \simeq 1 / t_{\text {eq }}$

$$
\frac{d \Omega_{H}(k)}{d \log k}=\frac{d \rho_{H}}{d \log k} \rho_{\mathrm{rad}}^{-1} \Omega_{\mathrm{rad}} \simeq r \times 10^{-10} \Omega_{\mathrm{rad}}, \quad k>\mathscr{H}_{\mathrm{eq}} .
$$

The ratio $r$ denotes the tensor to scalar ratio of the CMB anisotropy spectrum, see Section 3.2.

\subsubsection{Nonrelativistic matter, dust}

We now consider the case of nonrelativistic particles, dust, where we may neglect pressure, $w=c_{s}^{2}=0$. To simplify the equations we also neglect curvature, $K=0$. Then $a \propto t^{2}$ and the Bardeen equation then reduces to

$$
\ddot{\Psi}+\frac{6}{t} \dot{\Psi}=0
$$

with solution

$$
\Psi=A+\frac{B}{(k t)^{5}} .
$$

The second term is decaying and can therefore soon be neglected so that $\Psi=A \equiv \Psi_{0}$ is constant. The momentum conservation equation now yields

$$
\dot{V}+\frac{2}{t} V=k A
$$

which is solved by

$$
V(t)=\frac{A}{3} k t+C(k t)^{-2} .
$$

Again, the second term describes a decaying solution which we neglect. From

$$
\dot{D}_{g}+k V=0
$$

we find

$$
D_{g}=-\frac{A}{6}(k t)^{2}+A^{\prime}
$$

Here we cannot neglect the constant mode $A^{\prime}$ since it dominates on very large scales, $k t \ll 1$. Inserting the solution in the (00) Einstein equation,

$$
D=D_{g}+3 k^{-1} \mathscr{H} V+3 \Psi=-\frac{k^{2}}{4 \pi G a^{2} \rho} \Psi=-\frac{1}{6}(k t)^{2} \Psi,
$$

one finds $A^{\prime}=-5 A$. We therefore end up with

$$
\begin{aligned}
\Psi & =A=\Psi_{0}=\text { const. } \\
V & =\frac{1}{3} \Psi_{0} k t \\
D_{g} & =-5 \Psi_{0}-\frac{1}{6} \Psi_{0}(k t)^{2}, \quad D=-\frac{1}{6} \Psi_{0}(k t)^{2} .
\end{aligned}
$$


The metric perturbation $\Psi$ stays constant, it does not grow. This led Lifshitz, who was the first to investigate relativistic cosmological perturbation theory, to the conclusion that galaxies cannot have grown out of small initial fluctuations by gravitational instability [13]. However, as we see from Eq. (2.93), density perturbations do grow and therefore, on sufficiently small scales, $k \gg \mathscr{H}$, gravitational collapse is possible. Nevertheless, the fluctuations in the CMB which, as we shall see, reflect the amplitude of the gravitational potential $\Psi$, are of the order of the initial fluctuations generated during inflation.

\subsubsection{Radiation}

We now consider a spatially flat, radiation dominated universe, $w=c_{s}^{2}=1 / 3$ and $K=0$ so that $a \propto t$ and $\mathscr{H}=1 / t$. The Bardeen equation reduces to

with solution

$$
\ddot{\Psi}+\frac{4}{t} \dot{\Psi}+\frac{k^{2}}{3} \Psi=0
$$

$$
\Psi=\frac{\sqrt{3}}{k t}\left[A j_{1}\left(\frac{k t}{\sqrt{3}}\right)+B y_{1}\left(\frac{k t}{\sqrt{3}}\right)\right] .
$$

Here $j_{1}$ and $y_{1}$ are spherical Bessel functions, see e.g. [12]. Since the $y_{1}$-mode is singular for $t \rightarrow 0$, we have to set $B=0$. To determine the density and velocity perturbations, we use energy conservation and the Poisson equation (00 Einstein eq.) for radiation. Defining $x=k t / \sqrt{3}$ and ${ }^{\prime}=\frac{d}{d x}$ these become

$$
\begin{aligned}
D_{g}^{\prime} & =-\frac{4}{\sqrt{3}} V, \\
-2 x^{2} \Psi & =D_{g}+4 \Psi+\frac{4}{\sqrt{3} x} V .
\end{aligned}
$$

Inserting the solution (2.95) with $B=0$ for $\Psi$, we obtain

$$
\begin{aligned}
D_{g} & =2 A\left[\cos (x)-\frac{2}{x} \sin (x)\right], \\
V & =-\frac{\sqrt{3}}{4} D_{g}^{\prime} \\
\Psi & =-\frac{D_{g}+\frac{4}{\sqrt{3} x} V}{4+2 x^{2}}
\end{aligned}
$$

For Eq. (2.98) we have used the explicite form of the spherical Bessel function $j_{1}(x)=x^{-2} \sin (x)-$ $x^{-1} \cos (x)$.

In the super-horizon regime, $x \ll 1$, this yields

$$
\Psi=\frac{A}{3}, \quad D_{g}=-2 A\left(1+\frac{1}{3} x^{2}\right), \quad V=\frac{A}{2 \sqrt{3}} x .
$$

On sub-horizon scales, $x \gg 1$, we find oscillating solutions with constant amplitude and with frequency $k / \sqrt{3}$ for the density and velocity perturbations and an oscillating and decaying gravitational potential,

$$
\begin{aligned}
V & =\frac{\sqrt{3} A}{2} \sin (x), \\
D_{g} & =2 A \cos (x), \quad \Psi=-A \cos (x) / x^{2} .
\end{aligned}
$$


The radiation fluid cannot simply 'collapse' under gravity. Like for acoustic waves, the restoring force provided by the pressure leads to oscillations with constant amplitude. These are called the 'acoustic oscillations' of the radiation fluid. As we shall see in the next section, they are responsible for the acoustic peaks in the CMB fluctuation spectrum.

Also for radiation perturbations

$$
D=-\frac{A}{3 \sqrt{3}} x^{2} \ll \Psi
$$

is small on super horizon scales, $x \ll 1$.

The perturbation amplitude is given by the largest gauge-invariant perturbation variable. We conclude therefore that perturbations outside the Hubble horizon are frozen to first order. Once they enter the horizon they start to collapse, but pressure resists the gravitational force and the radiation fluid fluctuations oscillate at constant amplitude. The perturbations of the gravitational potential oscillate and decay like $1 / a^{2}$ inside the horizon.

\section{CMB anisotropies and polarization}

\subsection{Lightlike geodesics and CMB anisotropies}

After decoupling, $t>t_{\mathrm{dec}}$, photons follow to a good approximation light-like geodesics. The temperature shift of a Planck distribution of photons is equal to the energy shift of any given photon. The relative energy shift, $\Delta \omega / \omega$, (red- or blueshift) is independent of the photon energy (gravity is 'achromatic').

The unperturbed photon trajectory is given by

$$
\left(x^{\mu}(t)\right) \equiv\left(t,-\int_{t}^{t_{0}} \mathbf{n}\left(t^{\prime}\right) d t^{\prime}+\mathbf{x}_{0}\right),
$$

where $\mathbf{x}_{0}$ is the photon position at time $t_{0}$ and $\mathbf{n}$ is the (parallel transported) photon direction, proportional to the momentum of the photon. We determine the components of the photon momentum with respect to a geodesic basis $\left(\mathbf{e}_{i}\right)_{i=1}^{3}$ on the constant time hypersurfaces. We choose

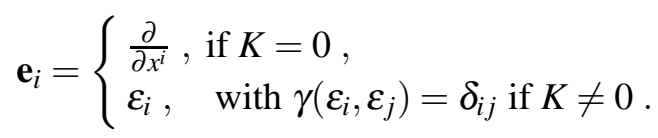

In other words, the vector fields $\varepsilon_{i}$ form an orthonormal basis for the spatial metric $\gamma_{i j}$.

Our perturbed metric is of the form

$$
\begin{aligned}
& d \tilde{s}^{2}=a^{2} d s^{2}, \quad \text { with } \\
& d s^{2}=\left(\gamma_{\mu v}+h_{\mu v}\right) d x^{\mu} d x^{v}, \quad \gamma_{00}=-1, \gamma_{i 0}=0, \gamma_{i j}=\gamma_{j i} .
\end{aligned}
$$

We make use of the fact that light-like geodesics are conformally invariant. More precisely, $d s^{2}$ and $d \tilde{s}^{2}$ have the same light-like geodesics, only the corresponding affine parameters are different. Let us denote the two affine parameters by $\lambda$ and $\tilde{\lambda}$ respectively, and the tangent vectors to the unperturbed geodesic by

$$
n=\frac{d x}{d \lambda}, \quad \tilde{n}=\frac{d x}{d \tilde{\lambda}}, \quad n^{2}=\tilde{n}^{2}=0, n^{0}=1, \mathbf{n}^{2}=1 .
$$


The photon 4-momentum $p^{\mu}$ is then given by $p^{\mu}=\omega n^{m} u$, where $\omega$ is the constant energy of the photon moving in the metric $d s^{2}$. We have seen that in expanding space the photon momentum is redshifted. Actually, the components behave like $\tilde{n}^{i} \propto 1 / a^{2}$ so that $\tilde{\mathbf{n}}^{2}=a^{2} \sum_{i}\left(\tilde{n}^{i}\right)^{2} \propto 1 / a^{2}$, hence we have to choose $\tilde{\lambda}=a^{2} \lambda$. As always for lightlike geodesics, $\tilde{\lambda}$ and $\lambda$ are only determined up to a multiplicative constant which we have fixed by the conditions $\mathbf{n}^{2}=1$ and $\tilde{\lambda}=a^{2} \lambda$.

Let us now introduce perturbations $\delta n^{\mu}$. For example, we set $n^{0}=1+\delta n^{0}$. The geodesic equation for the perturbed metric

$$
d s^{2}=\left(\gamma_{\mu v}+h_{\mu v}\right) d x^{\mu} d x^{v}
$$

yields, to first order,

$$
\frac{d}{d \lambda} \delta n^{\mu}=-\delta \Gamma_{\alpha \beta}^{\mu} n^{\alpha} n^{\beta}
$$

For the energy shift, we have to determine $\delta n^{0}$. Since $g^{0 \mu}=-\delta_{0 \mu}+$ first order, we obtain $\delta \Gamma_{\alpha \beta}^{0}=$ $-\frac{1}{2}\left(h_{\alpha 0 \mid \beta}+h_{\beta 0 \mid \alpha}-\dot{h}_{\alpha \beta}\right)$, so that

$$
\frac{d}{d \lambda} \delta n^{0}=h_{\alpha 0 \mid \beta} n^{\beta} n^{\alpha}-\frac{1}{2} \dot{h}_{\alpha \beta} n^{\alpha} n^{\beta}
$$

Integrating this equation we use $h_{\alpha 0 \mid \beta} n^{\beta} n^{\alpha}=\frac{d}{d \lambda}\left(h_{\alpha 0} n^{\alpha}\right)$, so that the change of $n^{0}$ between some initial time $t_{i}$ and some final time $t_{f}$ is given by

$$
\left.\delta n^{0}\right|_{i} ^{f}=\left[h_{00}+h_{0 j} n^{j}\right]_{i}^{f}-\frac{1}{2} \int_{i}^{f} \dot{h}_{\mu v} n^{\mu} n^{v} d \lambda .
$$

The energy of a photon with 4-momentum $\tilde{p}^{\mu}$ as seen by an observer moving with 4-velocity $\tilde{u}$ is

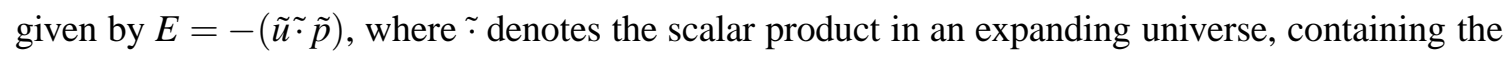
factor $a^{2}$. Hence, the ratio of the energy of a photon measured by some observer at $t_{f}$ to the energy emitted at $t_{i}$ is

$$
\frac{E_{f}}{E_{i}}=\frac{(\tilde{n} \cdot \tilde{u})_{f}}{(\tilde{n} \cdot \tilde{u})_{i}}=\frac{a_{i}}{a_{f}} \frac{(n \cdot u)_{f}}{(n \cdot u)_{i}} .
$$

Here $\tilde{u}$ is the emitter and receiver four-velocity in an expanding universe, $\tilde{u}=a^{-1} u$ while $u_{f}$ and $u_{i}$ are the four-velocities of the observer and emitter respectively in the non-expanding conformally related geometry given by

$$
u=(1-A) \partial_{t}+v^{i} \mathbf{e}_{i}=a \tilde{u} .
$$

Together with $\tilde{n}=a^{-2} n$ this implies the result (3.9). The ratio $a_{i} / a_{f}=T_{i} / T_{f}$ is the usual (unperturbed) redshift which relates $n$ and $\tilde{n}$. An observer measuring a temperature $T_{0}$ receives photons that were emitted at the time $t_{\mathrm{dec}}$ of decoupling of matter and radiation, at the fixed temperature $T_{\mathrm{dec}}$. In first-order perturbation theory, we find the following relation between the unperturbed temperatures $T_{f}, T_{i}$, the true temperatures $T_{0}=T_{f}+\delta T_{f}, T_{\mathrm{dec}}=T_{i}+\delta T_{i}$, and the photon density perturbation:

$$
\frac{a_{i}}{a_{f}}=\frac{T_{f}}{T_{i}}=\frac{T_{0}}{T_{\mathrm{dec}}}\left(1-\frac{\delta T_{f}}{T_{f}}+\frac{\delta T_{i}}{T_{i}}\right)=\frac{T_{0}}{T_{\mathrm{dec}}}\left(1-\left.\frac{1}{4} \delta_{r}\right|_{i} ^{f}\right),
$$

where $\delta_{r}$ is the intrinsic density perturbation in the radiation and we have used $\rho_{r} \propto T^{4}$ in the last equality. Inserting the above equation and Eq. (3.8) into Eq. (3.9), and using Eq. (2.22) for the 
definition of $h_{\mu \nu}$, as well as Eqs. $(2.30,2.31,2.57)$ and (2.54) one finds, after integration by parts, the following result for scalar perturbations [14]:

$$
\frac{E_{f}}{E_{i}}=\frac{T_{0}}{T_{\mathrm{dec}}}\left\{1-\left[\frac{1}{4} D_{g}^{(r)}+V_{j}^{(b)} n^{j}+\Psi+\Phi\right]_{i}^{f}+\int_{i}^{f}(\dot{\Psi}+\dot{\Phi}) d \lambda\right\} .
$$

Here $D_{g}^{(r)}$ denotes the density perturbation in the radiation fluid, and $V^{(b)}$ is the peculiar velocity of the baryonic matter component (the emitter and observer of radiation).

Evaluating Eq. (3.12) at final time $t_{0}$ (today) and initial time $t_{\mathrm{dec}}$, we obtain the temperature difference of photons coming from different directions $\mathbf{n}_{1}$ and $\mathbf{n}_{2}$

$$
\frac{\Delta T}{T} \equiv \frac{\Delta T\left(\mathbf{n}_{1}\right)}{T}-\frac{\Delta T\left(\mathbf{n}_{2}\right)}{T} \equiv \frac{E_{f}}{E_{i}}\left(\mathbf{n}_{1}\right)-\frac{E_{f}}{E_{i}}\left(\mathbf{n}_{2}\right) .
$$

Direction independent contributions to $\frac{E_{f}}{E_{i}}$ do not enter in this difference.

The largest contribution to $\frac{\Delta T}{T}$ is the dipole term, $V_{j}^{(b)}\left(t_{0}\right) n^{j}$ which simply describes our motion with respect to the emission surface. Its amplitude is about $1.2 \times 10^{-3}$ and it has been measured so accurately that even the yearly variation due to the motion of the earth around the sun has been detected [15].

For the higher multipoles (polynomials in $n^{j}$ of degree 2 and higher) we can set

$$
\frac{\Delta T(\mathbf{n})}{T}=\left[\frac{1}{4} D_{g}^{(r)}+V_{j}^{(b)} n^{j}+\Psi+\Phi\right]\left(t_{\mathrm{dec}}, \mathbf{x}_{d e c}\right)+\int_{t_{\mathrm{dec}}}^{t_{0}}(\dot{\Psi}+\dot{\Phi})(t, \mathbf{x}(t)) d t
$$

where $\mathbf{x}(t)$ is the unperturbed photon position at time $t$ for an observer at $\mathbf{x}_{0}$, and $\mathbf{x}_{\mathrm{dec}}=\mathbf{x}\left(t_{\mathrm{dec}}\right)$ (If $K=0$ we simply have $\mathbf{x}(t)=\mathbf{x}_{0}-\left(t_{0}-t\right) \mathbf{n}$.). The first term in Eq. (3.14) is the one we have discussed in the previous section. It describes the intrinsic inhomogeneities of the radiation density on the surface of last scattering, due to acoustic oscillations prior to decoupling, see Eq. (2.98). Depending on the initial conditions, it can contribute significantly also on super-horizon scales. This is especially important in the case of adiabatic initial conditions. One can show that (see [3]), in a dust + radiation universe with vanishing curvature, adiabatic initial conditions imply $D_{g}^{(r)}(k, t)=-\frac{20}{3} \Psi(k, t)$ and $V^{(b)}=V^{(r)} \ll D_{g}^{(r)}$ when $k t \ll 1$. With $\Phi=\Psi$ the square bracket of Eq. (3.14) therefore gives for adiabatic perturbations

$$
\left(\frac{\Delta T(\mathbf{n})}{T}\right)_{\text {adiabatic }}^{(O S W)}=\frac{1}{3} \Psi\left(t_{\mathrm{dec}}, \mathbf{x}_{\mathrm{dec}}\right)
$$

on super-horizon scales. The contribution to $\frac{\Delta T}{T}$ from the last scattering surface on very large scales is called the 'ordinary Sachs-Wolfe effect' (OSW). It has been derived for the first time by Sachs and Wolfe [16].

Apart from adiabatic initial conditions, a dust+radiation system can obey so called iso-curvature initial conditions which are defined by the requirement that $\Psi \rightarrow 0$ for $k t \rightarrow 0$. These initial conditions imply that $D_{g}^{(r)}(k, t) \rightarrow 0$ for $t \rightarrow 0$ so that the contribution of $D_{g}^{(r)}$ to the ordinary Sachs-Wolfe effect can be neglected,

$$
\left(\frac{\Delta T(\mathbf{n})}{T}\right)_{\text {iso-curvature }}^{(O S W)}=2 \Psi\left(t_{\mathrm{dec}}, \mathbf{x}_{\mathrm{dec}}\right) .
$$


The second term in (3.14) describes the relative motion of emitter and observer. This is the Doppler contribution to the CMB anisotropies. It appears on the same angular scales as the acoustic term ; we call the sum of the acoustic and Doppler contributions 'acoustic peaks'.

The integral in Eq. (3.14) accounts for red-shift or blue-shift caused by the time dependence of the gravitational potential along the path of the photon, and represents the so-called integrated Sachs-Wolfe (ISW) effect. In a $\Omega=1$, pure dust universe, as we have seen, the Bardeen potentials are constant and there is no integrated Sachs-Wolfe effect; the blue-shift which the photons acquire by falling into a gravitational potential is exactly canceled by the redshift induced by climbing out of it. This is no longer true in a universe with substantial radiation contribution, curvature, or a cosmological constant. The sum of the ordinary Sachs-Wolfe term and the integral is the full Sachs-Wolfe contribution (SW).

For vector perturbations $\delta^{(r)}$ and $A$ vanish and Eq. (3.9) leads to

$$
\left(\frac{E_{f}}{E_{i}}\right)^{(V)}=\frac{a_{i}}{a_{f}}\left[1-\left.V_{j}^{(b)} n^{j}\right|_{i} ^{f}+\int_{i}^{f} \sigma_{j} n^{j} d \lambda\right] .
$$

We obtain a Doppler term and a gravitational contribution. For tensor perturbations, i.e., gravitational waves, only the gravitational part remains,

$$
\left(\frac{E_{f}}{E_{i}}\right)^{(T)}=\frac{a_{i}}{a_{f}}\left[1-\int_{i}^{f} \dot{H}_{l j} n^{l} n^{j} d \lambda\right] .
$$

Equations (3.12), (3.15) and (3.16) are the manifestly gauge-invariant results for the energy shift of photons due to scalar, vector and tensor perturbations. Disregarding again the dipole contribution due to our proper motion, Eqs. $(3.15,3.16)$ imply the vector and tensor temperature fluctuations

$$
\begin{aligned}
& \left(\frac{\Delta T(\mathbf{n})}{T}\right)^{(V)}=V_{j}^{(b)}\left(t_{\mathrm{dec}}, \mathbf{x}_{\mathrm{dec}}\right) n^{j}+\int_{i}^{f} \sigma_{j}(t, \mathbf{x}(t)) n^{j} d \lambda, \\
& \left(\frac{\Delta T(\mathbf{n})}{T}\right)^{(T)}=-\int_{i}^{f} \dot{H}_{l j}(t, \mathbf{x}(t)) n^{l} n^{j} d \lambda .
\end{aligned}
$$

Note that for models where initial fluctuations have been laid down in the very early universe, vector perturbations are irrelevant as we have already pointed out. In this sense Eq. (3.17) is here mainly for completeness. However, in models where perturbations are sourced by some inherently inhomogeneous component (e.g. topological defects, see [17]), vector perturbations can be important.

\subsection{Power spectra}

\subsubsection{General comments}

The quantities which we can determine from a given model are usually not the precise values of perturbation variables like $\Psi(\mathbf{x}, t)$, but only expectation values like $\langle\Psi(\mathbf{x}, t) \Psi(\mathbf{y}, t)\rangle$. If we assume that the random process which generates the fluctuations $\Psi$ is stochastically homogeneous and isotropic, the correlator of the Fourier transform $\Psi(\mathbf{k}, t)$ has to vanish for different wave vectors $\mathbf{k}$. The quantity which we can calculate for a given model and which then has to be compared with observations is the power spectrum defined below. Power spectra are the 'harmonic transforms' 
of the two point correlation function ${ }^{1}$. If the perturbations of the model under consideration are Gaussian, a relatively generic prediction from inflationary models, then the two-point functions and therefore the power spectra contain the full statistical information of the model.

There is one additional problem to consider: one can never 'measure' expectation values. We have only one Universe, i.e., one realization of the stochastic process which generates the fluctuations at our disposal for observations. The best we can do when we want to determine the mean square fluctuation on a given scale $\lambda$ is to average over many disjoint patches of size $\lambda$, assuming that this spatial averaging corresponds to an ensemble averaging; a type of 'ergodic hypothesis'. This works well as long as the scale $\lambda$ is much smaller than the Hubble horizon, the size of the observable universe. For $\lambda \sim \mathscr{O}\left(H_{0}^{-1}\right)$ we can no longer average over many independent volumes and the value measured could be quite far from the ensemble average. This problem is known under the name 'cosmic variance'.

For an arbitrary scalar variable $X$ in position space, we define the power spectrum in Fourier space by

$$
\left\langle X\left(\mathbf{k}, t_{0}\right) X^{*}\left(\mathbf{k}^{\prime}, t_{0}\right)\right\rangle=(2 \pi)^{3} \delta\left(\mathbf{k}-\mathbf{k}^{\prime}\right) P_{X}(k) .
$$

In flat space, $K=0$, the function $X(\mathbf{k})$ is the ordinary Fourier transform of $X(\mathbf{x})$. If $K \neq 0$ the situation is more complicated. Then $X(\mathbf{k})$ represents an expansion of $X(\mathbf{x})$ in terms of eigenfunctions of the Laplacian and in the case $K>0$ the Dirac $\delta$-function has to be replaced by a discrete Kronecker $\delta$.

The \langle\rangle indicates a statistical average, ensemble average, over 'random initial conditions' in a given model. We assume that no point in space is preferred, in other words that $X(\mathbf{x})$ and any other stochastic field which we consider has the same distribution in every point $\mathbf{x}$. Such random fields are called 'statistically homogeneous' (or stationary). We further assume that the distribution of $X(\mathbf{x})$ has no preferred direction. This means that the random field $X$ is statistically isotropic. These properties imply that the Fourier transform of the 2-point function is diagonal, i.e., they explain the factor $\delta\left(\mathbf{k}-\mathbf{k}^{\prime}\right)$ in Eq. (3.19).

\subsubsection{The matter power spectrum}

Let us first consider the matter power spectrum, $P_{D}(k)$, which is defined by

$$
\left\langle D_{g m}\left(\mathbf{k}, t_{0}\right) D_{g m}^{*}\left(\mathbf{k}^{\prime}, t_{0}\right)\right\rangle=P_{D}(k)(2 \pi)^{3} \delta\left(\mathbf{k}-\mathbf{k}^{\prime}\right) .
$$

$P_{D}(k)$ is usually compared with the observed power spectrum of the galaxy distribution. This is clearly problematic since it is by no means evident what the relation between these two spectra should be. This problem is known under the name 'biasing' and it is very often simply assumed that the matter and galaxy power spectra differ only by a constant factor. The hope is also that on sufficiently large scales, since the evolution of both, galaxies and dark matter is governed by gravity, their power spectra should not differ by much. This hope seems to be reasonably justified. In [18] it is found that the observed galaxy power spectrum and the matter power spectrum inferred from the observation of CMB anisotropies differ only by about $10 \%$ on large scales.

\footnotetext{
${ }^{1}$ The 'harmonic transform' in usual flat space is simply the Fourier transform. In curved space it is the expansion in terms of eigenfunctions of the Laplacian on that space, e.g., on the sphere it corresponds to the expansion in terms of spherical harmonics.
} 
The power spectrum of velocity perturbations satisfies the relation

$$
\begin{aligned}
\left\langle V_{j}\left(\mathbf{k}, t_{0}\right) V_{i}^{*}\left(\mathbf{k}^{\prime}, t_{0}\right)\right\rangle & =Q_{j}^{(S)}(\mathbf{k}) Q_{i}^{(S) *}\left(\mathbf{k}^{\prime}\right) P_{V}(k)(2 \pi)^{3} \delta\left(\mathbf{k}-\mathbf{k}^{\prime}\right), \\
P_{V}(k) & \simeq H_{0}^{2} \Omega_{m}^{1.2} P_{D}(k) k^{-2}
\end{aligned}
$$

For $\simeq$ we have used that $\left|k V\left(t_{0}\right)\right|=\dot{D}_{g}^{(m)}\left(t_{0}\right) \sim H_{0} \Omega_{m}^{0.6} D_{g}$ on sub-horizon scales (see e.g., [19]) Here $\Omega_{m}$ is the matter density parameter. Unfortunately it is very difficult to measure peculiar velocities and so far not much use could be made of the velocity power spectrum.

\subsubsection{The CMB power spectrum}

Definition The spectrum in which we are most interested and which can be both, measured and calculated to the best accuracy is the CMB anisotropy power spectrum. It is defined as follows: $\Delta T / T$ is a function of position $\mathbf{x}$, time $t$ and photon direction $\mathbf{n}$. Here, i.e., at $\mathbf{x}=\mathbf{x}_{0}$ and now, i.e., at $t=t_{0}, \Delta T / T$ is a function on the sphere, $\mathbf{n} \in \mathbb{S}^{2}$. We develop it in terms of spherical harmonics, $Y_{\ell m}$ 's. We will often suppress the arguments $t_{0}$ and $\mathbf{x}_{0}$ in the following calculations. Since our fields are statistically homogeneous, averages over an ensemble of realizations (expectation values) are independent of position. Furthermore, we assume that the process generating the initial perturbations is statistically isotropic. This means that the distribution of $\Delta T / T(\mathbf{n})$ is the same for all directions $\mathbf{n}$. Like for Fourier transforms of random fields in space, this implies that the harmonic transform of $\Delta T / T$ is diagonal. On other words, the off-diagonal correlators of the expansion coefficients $a_{\ell m}$ vanish and we have

$$
\frac{\Delta T}{T}\left(t_{0}, \mathbf{x}_{0}, \mathbf{n}\right)=\sum_{\ell, m} a_{\ell m}\left(\mathbf{x}_{0}\right) Y_{\ell m}(\mathbf{n}), \quad\left\langle a_{\ell m} \cdot a_{\ell^{\prime} m^{\prime}}^{*}\right\rangle=\delta_{\ell \ell^{\prime}} \delta_{m m^{\prime}} C_{\ell} .
$$

The $C_{\ell}$ 's are the CMB power spectrum.

The two point correlation function is related to the $C_{\ell}$ 's by

$$
\begin{gathered}
\left\langle\frac{\Delta T}{T}(\mathbf{n}) \frac{\Delta T}{T}\left(\mathbf{n}^{\prime}\right)\right\rangle_{\mathbf{n} \cdot \mathbf{n}^{\prime}=\mu}=\sum_{\ell, \ell^{\prime}, m, m^{\prime}}\left\langle a_{\ell m} \cdot a_{\ell^{\prime} m^{\prime}}^{*}\right\rangle Y_{\ell m}(\mathbf{n}) Y_{\ell^{\prime} m^{\prime}}^{*}\left(\mathbf{n}^{\prime}\right)= \\
\sum_{\ell} C_{\ell} \underbrace{\sum_{m=-\ell}^{\ell} Y_{\ell m}(\mathbf{n}) Y_{\ell m}^{*}\left(\mathbf{n}^{\prime}\right)}_{\frac{2 \ell+1}{4 \pi} P_{\ell}\left(\mathbf{n} \cdot \mathbf{n}^{\prime}\right)}=\frac{1}{4 \pi} \sum_{\ell}(2 \ell+1) C_{\ell} P_{\ell}(\mu),
\end{gathered}
$$

where we have used the addition theorem of spherical harmonics for the last equality; the $P_{\ell}$ 's are the Legendre polynomials (see e.g. [12]).

Clearly the $a_{l m}$ 's from scalar, vector and tensor perturbations are uncorrelated,

$$
\left\langle a_{\ell m}^{(S)} a_{\ell^{\prime} m^{\prime}}^{(V)}\right\rangle=\left\langle a_{\ell m}^{(S)} a_{\ell^{\prime} m^{\prime}}^{(T)}\right\rangle=\left\langle a_{\ell m}^{(V)} a_{\ell^{\prime} m^{\prime}}^{(T)}\right\rangle=0 .
$$

Since vector perturbations decay, their contributions, the $C_{\ell}^{(V)}$, are negligible in models where initial perturbations have been laid down very early, e.g., after an inflationary period. Tensor perturbations are constant on super-horizon scales and perform damped oscillations once they enter the horizon. 
Scalar perturbations Let us first discuss in somewhat more detail scalar perturbations. We specialize to the case $K=0$ for simplicity. We suppose the initial perturbations to be given by a spectrum of the form

$$
\left\langle\Psi(\mathbf{k}) \Psi^{*}\left(\mathbf{k}^{\prime}\right)\right\rangle k^{3}=(2 \pi)^{3} k^{3} P_{\Psi}(k) \boldsymbol{\delta}\left(\mathbf{k}-\mathbf{k}^{\prime}\right)=(2 \pi)^{3} A_{S}\left(k t_{0}\right)^{n-1} \boldsymbol{\delta}\left(\mathbf{k}-\mathbf{k}^{\prime}\right) .
$$

We multiply by the constant $t_{0}^{n-1}$, the present comoving size of the horizon, in order to keep $A_{S}$ dimensionless for all values of $n$. The number $n$ is called the spectral index. $A_{S}$ then represents the amplitude of metric perturbations at horizon scale today, $k=1 / t_{0}$.

As we have seen in the previous section, the dominant contribution on super-horizon scales (neglecting the integrated Sachs-Wolfe effect $\int \dot{\Phi}+\dot{\Psi}$ ) is the ordinary Sachs-Wolfe effect, OSW, which for adiabatic perturbations is given by

$$
\frac{\Delta T}{T}\left(\mathbf{x}_{0}, \mathbf{n}, t_{0}\right) \simeq \frac{1}{3} \Psi\left(\mathbf{x}_{\mathrm{dec}}, t_{\mathrm{dec}}\right) .
$$

Since $\mathbf{x}_{\mathrm{dec}}=\mathbf{x}_{0}-\mathbf{n}\left(t_{0}-t_{\mathrm{dec}}\right)$, the Fourier transform of (3.27) gives

$$
\frac{\Delta T}{T}\left(\mathbf{k}, \mathbf{n}, t_{0}\right)=\frac{1}{3} \Psi\left(\mathbf{k}, t_{\mathrm{dec}}\right) \cdot e^{i \mathbf{k n}\left(t_{0}-t_{\mathrm{dec}}\right)} .
$$

Using the decomposition

$$
e^{i \mathbf{k n}\left(t_{0}-t_{\mathrm{dec}}\right)}=\sum_{\ell=0}^{\infty}(2 \ell+1) i^{\ell} j_{\ell}\left(k\left(t_{0}-t_{\mathrm{dec}}\right)\right) P_{\ell}(\widehat{\mathbf{k}} \cdot \mathbf{n}),
$$

where $j_{\ell}$ are the spherical Bessel functions; and using the addition theorem of spherical harmonics repeatedly, we find

$$
\begin{aligned}
& \left\langle\frac{\Delta T}{T}\left(\mathbf{x}_{0}, \mathbf{n}, t_{0}\right) \frac{\Delta T}{T}\left(\mathbf{x}_{0}, \mathbf{n}^{\prime}, t_{0}\right)\right\rangle_{\mathbf{n n}^{\prime}=\mu} \simeq \\
& \sum_{\ell} \frac{2 \ell+1}{4 \pi} P_{\ell}(\mu) \frac{2}{\pi} \int \frac{d k}{k}\left\langle\frac{1}{9}|\Psi|^{2}\right\rangle k^{3} j_{\ell}^{2}\left(k\left(t_{0}-t_{\mathrm{dec}}\right)\right) .
\end{aligned}
$$

Comparing this equation with Eq. (3.24) we obtain for adiabatic perturbations on large angular scales, $2 \leq \ell \ll \chi\left(t_{0}-t_{\mathrm{dec}}\right) / t_{\mathrm{dec}} \sim 100$ :

$$
C_{\ell}^{(S W)} \simeq C_{\ell}^{(O S W)} \simeq \frac{2}{9 \pi} \int_{0}^{\infty} \frac{d k}{k}\left\langle|\Psi|^{2}\right\rangle k^{3} j_{\ell}^{2}\left(k\left(t_{0}-t_{\mathrm{dec}}\right)\right) .
$$

The function $j_{\ell}^{2}\left(k\left(t_{0}-t_{\mathrm{dec}}\right)\right)$ peaks roughly at $k\left(t_{0}-t_{\mathrm{dec}}\right) \simeq k t_{0} \simeq \ell$. If $\Psi$ is a pure power law on large scales, $k t_{\text {dec }} \lesssim 1$ as in Eq. (3.26) and we set $k\left(t_{0}-t_{\text {dec }}\right) \sim k t_{0}$, the integral (3.30) can be performed analytically. For the ansatz (3.26) one finds

$$
C_{\ell}^{(S W)}=\frac{A_{S}}{9} \frac{\Gamma(3-n) \Gamma\left(\ell-\frac{1}{2}+\frac{n}{2}\right)}{2^{3-n} \Gamma^{2}\left(2-\frac{n}{2}\right) \Gamma\left(\ell+\frac{5}{2}-\frac{n}{2}\right)} \quad \text { for }-3<n<3 .
$$

Of special interest is the scale invariant or Harrison-Zel'dovich (HZ) spectrum, $n=1$ which is generically produced in inflationary models. It leads to

$$
\ell(\ell+1) C_{\ell}^{(S W)}=\frac{A_{S}}{9 \pi} \simeq\left\langle\left(\frac{\Delta T}{T}\left(\vartheta_{\ell}\right)\right)^{2}\right\rangle, \quad \vartheta_{\ell} \equiv \pi / \ell .
$$


This is precisely (within the accuracy of the experiment) the behavior observed by the DMR (Differential Microwave Radiometer) experiment aboard the satellite COBE [20]. In the mean time, the scalar spectral index has been determined more precisely with the WMAP (Wilkinson Microwave Anisotropy Probe) satellite [21]. The result is $n=0.95 \pm 0.02$.

Inflationary models predict very generically a nearly $\mathrm{HZ}$ spectrum with $n$ slightly less than 1. The DMR discovery and the WMAP confirmation have therefore been regarded as a great success, if not as a proof, of inflation. There are, however, other models like topological defects (see [17]), or certain string cosmology models [22] which also predict scale-invariant, i.e., Harrison Zel'dovich spectra of fluctuations. These models are outside the class investigated here, since in them perturbations are induced by seeds which evolve non-linearly in time. They are not simply led down as initial conditions for the fluid perturbations but typically affect the perturbations of a given wave length until it crosses the Hubble scale. This generically leads to iso-curvature perturbations which are not ruled out by present data since they do not show prominent acoustic peaks, see Fig. 1.

For iso-curvature perturbations, the main contribution on large scales comes from the integrated Sachs-Wolfe effect and (3.30) is replaced by

$$
C_{\ell}^{(I S W)} \simeq \frac{8}{\pi} \int \frac{d k}{k} k^{3}\left\langle\left|\int_{t_{\mathrm{dec}}}^{t_{0}} \dot{\Psi}(k, t) j_{\ell}^{2}\left(k\left(t_{0}-t\right)\right) d t\right|^{2}\right\rangle .
$$

Inside the horizon $\Psi$ is roughly constant (matter dominated). Using the ansatz (3.26) for $\Psi$ inside the horizon and setting the integral in $(3.33) \sim 2 \Psi(k, t=1 / k) j_{\ell}^{2}\left(k t_{0}\right)$, we obtain again (3.31), but with $A_{S}^{2} / 9$ replaced by $4 A_{S}^{2}$. For a fixed amplitude $A_{S}$ of perturbations, the Sachs-Wolfe temperature anisotropies coming from iso-curvature perturbations are therefore about six times larger than those coming from adiabatic perturbations (see Fig. 1).

On smaller scales, $\ell \gtrsim 100$, the contribution to $\Delta T / T$ is dominated by acoustic oscillations, the first two terms in Eq. (3.14). Instead of (3.33) we then obtain

$$
C_{\ell}^{(A C)} \simeq \frac{2}{\pi} \int_{0}^{\infty} \frac{d k}{k} k^{3}\left\langle\left|\frac{1}{4} D_{r}\left(k, t_{\mathrm{dec}}\right) j_{\ell}\left(k t_{0}\right)+V^{(r)}\left(k, t_{\mathrm{dec}}\right) j_{\ell}^{\prime}\left(k t_{0}\right)\right|^{2}\right\rangle .
$$

To remove the SW contribution from $D_{g}^{(r)}$ we have simply replaced it by $D_{r}$ which is much smaller than $\Psi$ on super-horizon scales and therefore does not contribute to the SW terms. On subhorizon scales $D_{r} \simeq D_{g}^{(r)}$ and $V_{r}$ are oscillating like sine or cosine waves depending on the initial conditions. Correspondingly the $C_{\ell}^{(A C)}$ will show peaks and minima. For adiabatic initial conditions $D_{g}^{(r)}$ and therefore also $D_{r}$ oscillate like a cosine. Its minima and maxima are at $k_{n} t_{\mathrm{dec}} / \sqrt{3}=n \pi$. Odd values of $n$ correspond to maxima, 'contraction peaks', while even numbers are minima, 'expansion peaks'.

These are the 'acoustic peaks' of the CMB anisotropies. Sometimes they are miss-leadingly called 'Doppler peaks' referring to an old misconception that the peaks would be due to the velocity term in the above formula. Actually the contrary is true. At maxima and minima of the density contrast, the velocity (being proportional to the derivative of the density) nearly vanishes.

The angle $\theta_{n}$ which subtends the scale $\lambda_{n}=\pi / k_{n}$ at the last scattering surface is determined by the angular diameter distance to the last scattering surface, $d_{A}\left(t_{\mathrm{dec}}\right)$ via the relation $\theta_{n}=\lambda_{n} / d_{A}\left(t_{\mathrm{dec}}\right)$. 
Expanding the temperature anisotropies in spherical harmonics, the angular scale $\theta_{n}$ corresponds (roughly) to the harmonic number

$$
\ell_{n} \simeq \pi / \theta_{n}=\pi d_{A}\left(t_{\mathrm{dec}}\right) / \lambda_{n}=d_{A}\left(t_{\mathrm{dec}}\right) k_{n}=n \sqrt{3} \pi d_{A}\left(t_{\mathrm{dec}}\right) / t_{\mathrm{dec}}
$$

For a flat matter dominated universe $d_{A}\left(t_{\mathrm{dec}}\right) \simeq t_{0}$ leading to $\ell_{n} \simeq 180 n$. This crude approximation deviates by about $15 \%$ from the precise numerical value, which depends with $d_{A}$ strongly on curvature but also on the Hubble parameter and on the cosmological constant. Furthermore, the peak positions depend on the sound speed of the radiation-baryon plasma which we have simply set to $c_{s}=1 / \sqrt{3}$ in this approximation. A detailed discussion of the parameter dependence of the peak positions can be found in [3]. Note, however, that the position of the first peak differs significantly for the iso-curvature mode, for which $D_{g}^{(r)}$ oscillates like a sine. For generic initial conditions, we would expect a mixture of the sine and cosine modes which leads to a displacement of the first peak. The observed CMB anisotropies are consistent with a purely adiabatic mode and require, at least, that the adiabatic mode dominates [23, 24].

For a flat universe, $\Omega=1$, the $n$-th peak therefore is placed at

$$
\ell_{n} \simeq k_{n} t_{0} \simeq n \pi \sqrt{3} \frac{t_{0}}{t_{\mathrm{dec}}}
$$

For a flat matter dominated universe we have $\frac{t_{0}}{t_{\mathrm{dec}}} \sim \sqrt{z_{\mathrm{dec}}} \sim 33.2$ which yields $\ell_{1} \sim 180$. Here we have used $z_{\mathrm{dec}} \sim 1100$. This approximation is not very good since the universe is not very well matter dominated at $t_{\mathrm{dec}}$. A somewhat more accurate estimate gives $\ell_{1} \sim 220$, in good agreement with the numerical value. Subsequent peaks are then given by $\ell_{n}=n \ell_{1}$.

Our discussion is only valid in flat space. In curved space the exponentials $\exp \left(i k\left(t_{0}-t_{\mathrm{dec}}\right)\right)$ have to be replaced with the harmonics of the curved spaces. For the positions of the peaks, this corresponds to replace $k_{n} t_{0}$ by $k_{n} \chi\left(t_{0}\right)$, hence by replacing $t_{0}$ by the comoving angular diameter distance to the last scattering surface. Instead of Eq. (3.36) we then obtain the following approximate relation for the peak positions,

$$
\ell_{n} \sim n \pi \sqrt{3} \frac{\chi\left(t_{0}\right)}{t_{\mathrm{dec}}} .
$$

For values of $\Omega$ close to unity this scales like $1 / \sqrt{\Omega}$.

On very small scales the acoustic peaks are damped by the photon diffusion which takes place during the recombination process. This effect must be described with the Boltzmann equation approach (see next section and [3]).

Tensor perturbations For gravitational waves a formula analogous to (3.31) can be derived (see [3]),

$$
C_{\ell}^{(T)}=\frac{2}{\pi} \int d k k^{2}\left\langle\left|\int_{t_{\mathrm{dec}}}^{t_{0}} d t \dot{H}^{(T)}(t, k) \frac{j_{\ell}\left(k\left(t_{0}-t\right)\right)}{\left(k\left(t_{0}-t\right)\right)^{2}}\right|^{2}\right\rangle \frac{(\ell+2) !}{(\ell-2) !} .
$$

To a crude approximation we may assume $\dot{H}^{(T)}=0$ on super-horizon scales and

$$
\int d t \dot{H}^{(T)} j_{\ell}\left(k\left(t_{0}-t\right)\right) \sim H^{(T)}(t=1 / k) j_{\ell}\left(k t_{0}\right) .
$$




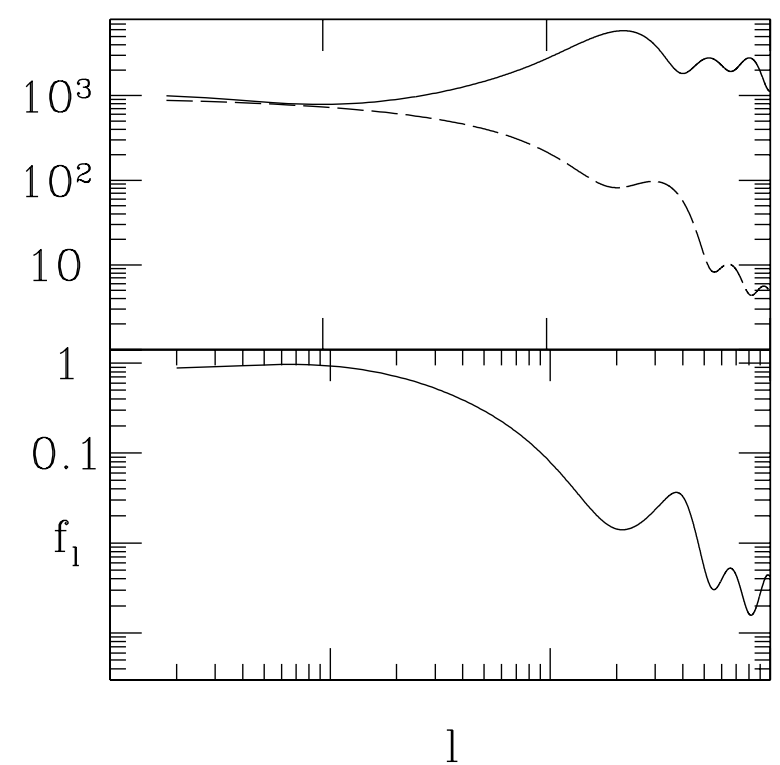

Figure 1: Examples of COBE normalized adiabatic (solid line) and iso-curvature (dashed line) CMB anisotropy spectra, $\ell(\ell+1) C_{\ell} /(2 \pi)$ in units of $(\mu K)^{2}$ are shown on the top panel. In the bottom panel the ratio of the iso-curvature to adiabatic temperature fluctuations is plotted.

For a pure power law,

$$
k^{3}\left\langle\left|H^{(T)}(k, t=1 / k)\right|^{2}\right\rangle=A_{T}\left(k t_{0}\right)^{n_{T}},
$$

one then obtains

$$
\begin{aligned}
C_{\ell}^{(T)} & \simeq \frac{2}{\pi} \frac{(\ell+2) !}{(\ell-2) !} A_{T} \int_{0}^{\infty} \frac{d x}{x} x^{n_{T}} \frac{j_{\ell}^{2}(x)}{x^{4}} \\
& =\frac{(\ell+2) !}{(\ell-2) !} A_{T} \frac{\Gamma\left(6-n_{T}\right) \Gamma\left(\ell-2+\frac{n_{T}}{2}\right)}{2^{6-n_{T}} \Gamma^{2}\left(\frac{7}{2}-n_{T}\right) \Gamma\left(\ell+4-\frac{n_{T}}{2}\right)} .
\end{aligned}
$$

For a scale invariant spectrum $\left(n_{T}=0\right)$ this results in

$$
\ell(\ell+1) C_{\ell}^{(T)} \simeq \frac{8}{15 \pi} \frac{\ell(\ell+1)}{(\ell+3)(\ell-2)} A_{T} .
$$

The singularity at $\ell=2$ in this crude approximation is not real, but there is some enhancement of $\ell(\ell+1) C_{\ell}^{(T)}$ for $\ell \lesssim 10$ (see Fig. 2).

Since tensor perturbations decay on sub-horizon scales, $\ell \gtrsim 100$, they are not very sensitive to cosmological parameters. Again, inflationary models (and topological defects) predict a scale invariant spectrum of tensor fluctuations $\left(n_{T} \sim 0\right)$.

Comparing the tensor and scalar result for scale invariant perturbations we obtain for large scales, $\ell<50$

$$
\frac{C_{\ell}^{(T)}}{C_{\ell}^{(S)}} \simeq \frac{72}{15} \frac{A_{T}}{A_{S}} \equiv r
$$


Present CMB anisotropy data favor a roughly scale invariant spectrum with amplitude

$$
\ell(\ell+1) C_{\ell} \simeq 6 \times 10^{-10} \text { for } \ell \lesssim 50 .
$$

If the perturbations are purely scalar, this requires $A_{S} \simeq 1.7 \times 10^{-8}$, if they were purely tensorial (which we know they are not), we would need $A_{T} \simeq 3.5 \times 10^{-9}$. In general observations require

$$
\frac{A_{S}}{9 \pi}(1+r) \simeq 6 \times 10^{-10}
$$

On small angular scales, $\ell \gtrsim 800$, fluctuations are damped by collisional damping (Silk damping). This effect has to be discussed with the Boltzmann equation for photons which is presented in the next section. 


\section{Scalar}
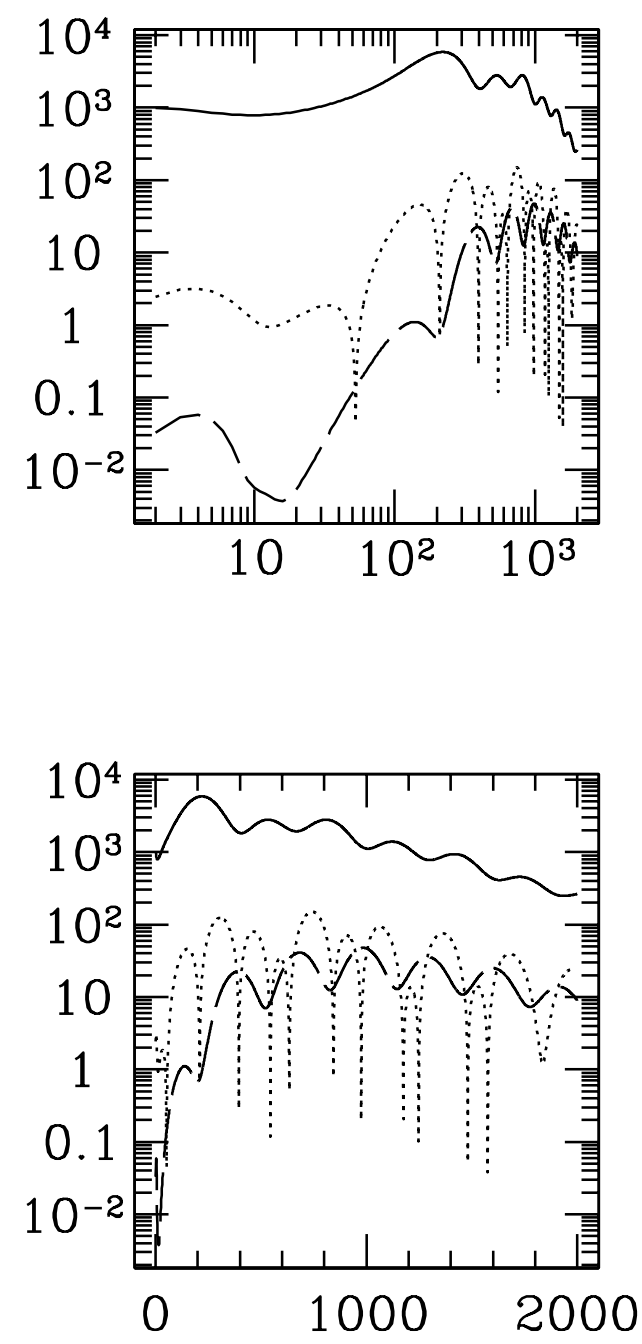

Tensor
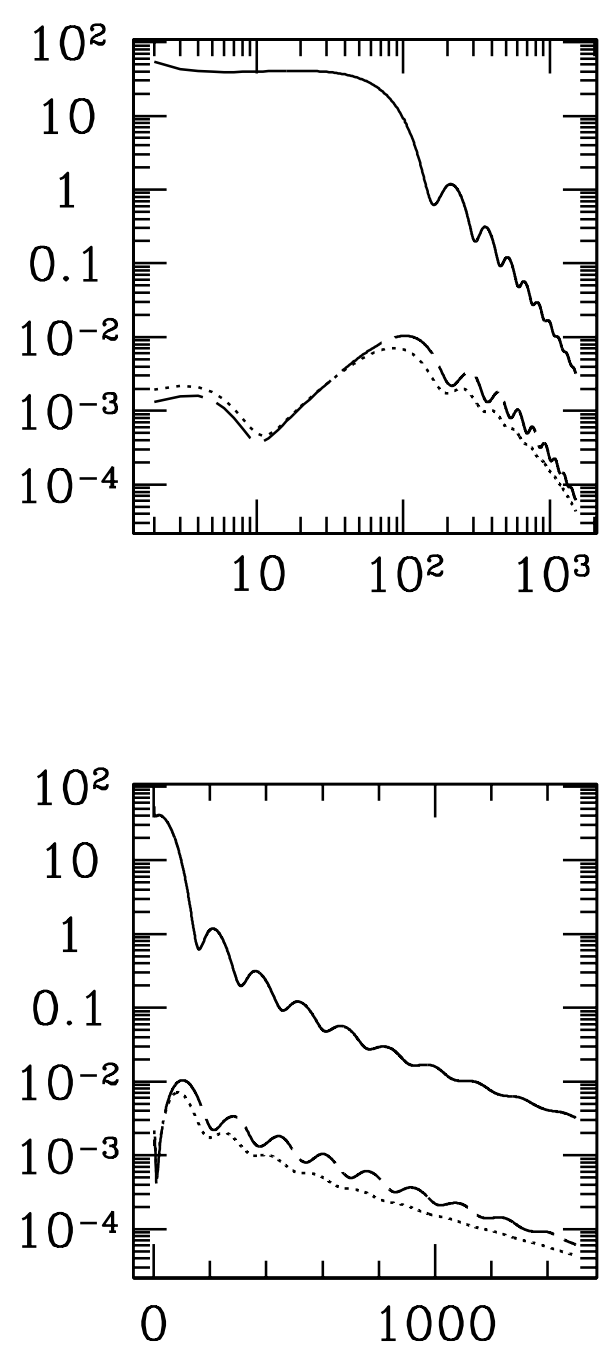

Figure 2: Adiabatic scalar and tensor CMB anisotropy spectra are plotted, $\ell(\ell+1) C_{\ell} /(2 \pi)$ in units of $(\mu K)^{2}$ in log-scale (top panels), where the Sachs Wolfe plateau is clearly visible and in linear scale (bottom panels) which shows the equal spacing of the acoustic peaks. The solid line shows the temperature spectrum, the dashed line is the polarization and the dotted line shows the temperature-polarization cross correlation (see next section). The latter can become negative, the deep spikes in the dotted curves in the left hand panels are actually sign changes. The left hand side shows scalar fluctuation spectra, while the right hand side shows tensor spectra. The observational data are well fitted by a purely scalar spectrum. Comparison of data and a model scalar spectrum are shown in Figs. 5 to 7. 


\subsection{The Boltzmann equation}

Photons (or any kind of "classical particle") can be described by the distribution function $f$ defined on the 7-dimensional phase space, the mass shell

$$
P_{m} \equiv\left\{(x, p) \in T \mathscr{S} \mid g_{\mu v}(x) p^{\mu} p^{v}=-m^{2}\right\} .
$$

Here $\mathscr{S}$ is the spacetime manifold and $T \mathscr{S}$ is the tangent space. For photons $m=0$. The energy momentum tensor is given by integrals of the second moments over the 'fiber'

$$
P_{m}(x) \equiv\left\{p \in T_{x} \mathscr{S} \mid g_{\mu v}(x) p^{\mu} p^{v}=-m^{2}\right\} .
$$

Here $T_{x} \mathscr{S}$ is the tangent space of $\mathscr{S}$ at the point $x \in \mathscr{M}$.

$$
f: P_{m} \rightarrow \mathbb{R}:(x, p) \mapsto f(x, p)
$$

is the one particle distribution function and the energy momentum tensor of the particles is given by

$$
T^{\mu v}(x)=\int_{P_{m}(x)} \frac{\sqrt{|g(x)|}}{\left|p_{0}(x, \mathbf{p})\right|} p^{\mu} p^{v} f(x, \mathbf{p}) d^{3} p .
$$

If the particles are not interacting (collisionless) they move along geodesics,

$$
\ddot{x}^{\mu}+\Gamma_{v \alpha}^{\mu} \dot{x}^{\nu} \dot{x}^{\alpha}=0 .
$$

The dot denotes the derivative with respect to proper time $s$ defined by the condition $g_{\mu v}(x) \dot{x}^{\mu} \dot{x}^{\nu}=$ $\dot{x}^{2}=-1$. In the case of massless (lightlike) particles, the proper time is only defined up to a multiplicative constant. For the distribution function this implies the Liouville equation,

$$
\frac{d f}{d t}=\left[p^{\mu} \partial_{\mu}-\Gamma_{\mu \nu}^{i} p^{\mu} p^{\nu} \frac{\partial}{\partial p^{i}}\right] f=0 .
$$

If there are collisions, the zero on the right hand side has to be replaced by the Boltzmann collision integral.

In an unperturbed Friedmann universe the Liouville equation just implies that physical momenta are redshifted, $p=\sqrt{g_{i j} p^{i} p^{j}} \propto 1 / a$. Hence the comoving momenta scale as $p^{i} \propto 1 / a^{2}$. Setting $v=a p$ and interpreting $f$ as function of $t$ and $v$ only, the Liouville equation on a Friedmann universe reduces to $\partial_{t} f=0$. Hence $f=f(v)$ is a function of the redshift corrected momenta only.

We now define $f(v, t, \mathbf{n}, \mathbf{x})=\bar{f}(v)+\delta f(v, t, \mathbf{n}, \mathbf{x})$ and

$$
\mathscr{M}(t, \mathbf{n}, \mathbf{x}) \equiv \frac{\pi}{\bar{\rho}_{\gamma} a^{4}} \int v^{4} \delta f d v \equiv \frac{1}{4} \frac{\delta \rho_{\gamma}(t, \mathbf{n}, \mathbf{x})}{\rho_{\gamma}} \equiv \frac{\Delta T}{T}(t, \mathbf{n}, \mathbf{x}) .
$$

After a lengthy derivation which is presented in [3], the Liouville equation for scalar perturbations then becomes

$$
\partial_{t} \mathscr{M}^{(S)}+n^{i} \partial_{i} \mathscr{M}^{(S)}=-n^{j}\left[\Psi_{, j}+\Phi_{, j}\right] .
$$

This equation can be solved formally for any given source term $\Phi+\Psi$. One easily checks that the solution with initial condition $\mathscr{M}^{(S)}\left(t_{\text {in }}, \mathbf{x}, \mathbf{n}\right)$ is

$$
\begin{aligned}
\mathscr{M}^{(S)}(t, \mathbf{x}, \mathbf{n})= & \mathscr{M}^{(S)}\left(t_{\mathrm{in}}, \mathbf{x}-\mathbf{n}\left(t-t_{\mathrm{in}}\right), \mathbf{n}\right) \\
& -\int_{t_{\mathrm{in}}}^{t} d t^{\prime} n^{i} \partial_{i}(\Psi+\Phi)\left(t^{\prime}, \mathbf{x}-\mathbf{n}\left(t-t^{\prime}\right)\right) .
\end{aligned}
$$


Using

$$
\begin{aligned}
\frac{d}{d t^{\prime}}(\Psi+\Phi)\left(t^{\prime}, \mathbf{x}-\mathbf{n}\left(t-t^{\prime}\right)\right)= & \partial_{t^{\prime}}(\Psi+\Phi)\left(t^{\prime}, \mathbf{x}-\mathbf{n}\left(t-t^{\prime}\right)\right) \\
& +n^{i} \partial_{i}(\Psi+\Phi)\left(t^{\prime}, \mathbf{x}-\mathbf{n}\left(t-t^{\prime}\right)\right),
\end{aligned}
$$

we can replace the second term on the right hand side to obtain

$$
\begin{aligned}
& \mathscr{M}^{(S)}(t, \mathbf{x}, \mathbf{n})= \mathscr{M}^{(S)}\left(t_{\text {in }}, \mathbf{x}-\mathbf{n}\left(t-t_{\text {in }}\right), \mathbf{n}\right) \\
&+(\Psi+\Phi)\left(t_{\text {in }}, \mathbf{x}-\mathbf{n}\left(t-t_{\text {in }}\right)\right) \\
&+\int d t^{\prime} \partial_{t^{\prime}}(\Psi+\Phi)\left(t^{\prime}, \mathbf{x}-\mathbf{n}\left(t-t^{\prime}\right)\right)+\text { monopole }
\end{aligned}
$$

By 'monopole' we denote the uninteresting $\mathbf{n}$-independent contribution $-(\Psi+\Phi)(t, \mathbf{x})$ which does not affect the CMB anisotropy spectrum. The Bardeen potentials $\Psi$ and $\Phi$, however, are given via Einstein's equation in terms of the perturbations of the energy momentum tensor which contain contributions from the photons which are in turn the momentum integrals of $\mathscr{M}$ given below. Therefore, even though it might look like it, this is not a solution of the Liouville equation. The term on the right hand side also depends on $\mathscr{M}^{(S)}$.

Let us compare Eq. (3.53) with the result from the integration of lightlike geodesics after decoupling in Eqs.(3.12) and (3.14). Here we have solved the Liouville equation which also does not take into account the scattering of photons and is therefore equivalent to our approach in the previous section. They both correspond to the 'sudden decoupling' approximation, where we assume that photons behaved like a perfect fluid before decoupling and were entirely free after decoupling. This is a relatively good approximation for all scales which are much larger than the duration of the process of recombination which correspond to multipoles $\ell \gtrsim 800$. The comparison with Eqs.(3.12) and (3.14) yields

$$
\mathscr{M}^{(S)}\left(t_{\mathrm{dec}}, \mathbf{x}-\mathbf{n}\left(t-t_{\mathrm{dec}}\right), \mathbf{n}\right)=\left(\frac{1}{4} D_{g}+\mathbf{n} \cdot \mathbf{V}^{(b)}\right)\left(t_{\mathrm{dec}}, \mathbf{x}-\mathbf{n}\left(t-t_{\mathrm{dec}}\right)\right),
$$

and

$$
\mathscr{M}^{(S)}(t, \mathbf{x}, \mathbf{n}) \equiv \frac{\delta T}{T}(t, \mathbf{x}, \mathbf{n}) .
$$

In Fourier space equation (3.52) becomes

$$
\mathscr{M}(t, \mathbf{k}, \mathbf{n})=e^{-i k \mu\left(t-t_{\mathrm{in}}\right)} \mathscr{M}\left(t_{\mathrm{in}}, \mathbf{k}, \mathbf{n}\right)+\int_{t_{\mathrm{in}}}^{t} d t^{\prime} e^{-i k \mu\left(t-t^{\prime}\right)} i k \mu(\Phi+\Psi)(\mathbf{k}, t), \quad \mu=\mathbf{n} \cdot \hat{\mathbf{k}} .
$$

\subsection{Polarization}

A photon with momentum $p \mathbf{n}$ is an electromagnetic wave propagating in direction $\mathbf{n}$. We define the polarization basis $\boldsymbol{\varepsilon}^{(1)}, \boldsymbol{\varepsilon}^{(2)}$, such that $\left(\boldsymbol{\varepsilon}^{(1)}, \boldsymbol{\varepsilon}^{(2)}, \mathbf{n}\right)$ form a right handed orthonormal system. Transversality of the electric field then requires that

$$
\mathbf{E}=E_{1} \boldsymbol{\varepsilon}^{(1)}+E_{2} \boldsymbol{\varepsilon}^{(2)} .
$$

The polarization tensor is defined by $E_{a} E_{b}^{*}$. This is a hermitian $2 \times 2$ matrix and can therefore be written as

$$
E_{a} E_{b}^{*}=\frac{1}{2}\left[I \sigma_{a b}^{(0)}+U \sigma_{a b}^{(1)}+V \sigma_{a b}^{(2)}+Q \sigma_{a b}^{(3)}\right]=\frac{1}{2} I \sigma_{a b}^{(0)}+P_{a b},
$$


where $\sigma^{(\alpha)}$ denote the Pauli matrices and the four real functions of the photon direction $\mathbf{n}, I, U, V$ and $Q$ are the Stokes parameters.

$$
\begin{aligned}
\sigma^{(0)}=\left(\begin{array}{ll}
1 & 0 \\
0 & 1
\end{array}\right), & \sigma^{(1)}=\left(\begin{array}{ll}
0 & 1 \\
1 & 0
\end{array}\right), \\
\sigma^{(2)}=\left(\begin{array}{cc}
0 & -i \\
i & 0
\end{array}\right), & \sigma^{(3)}=\left(\begin{array}{cc}
1 & 0 \\
0 & -1
\end{array}\right) .
\end{aligned}
$$

In terms of the electric field, the Stokes parameters are

$$
\begin{aligned}
I & =\left|E_{1}\right|^{2}+\left|E_{2}\right|^{2}, \quad Q=\left|E_{1}\right|^{2}-\left|E_{2}\right|^{2}, \\
U & =\left(E_{1}^{*} E_{2}+E_{2}^{*} E_{1}\right)=2 \operatorname{Re}\left(E_{1}^{*} E_{2}\right), \quad V=2 \operatorname{Im}\left(E_{1}^{*} E_{2}\right) .
\end{aligned}
$$

$I$ is simply the intensity of the electromagnetic wave. $Q$ represents the amount of linear polarization in directions $\boldsymbol{\varepsilon}^{(1)}$ and $\boldsymbol{\varepsilon}^{(2)}$, i.e., $Q$ is the difference between the intensity of radiation polarized along $\boldsymbol{\varepsilon}^{(1)}$ and the intensity polarized in direction $\boldsymbol{\varepsilon}^{(2)}$. The parameters $Q$ and $U$ describe the symmetric traceless part of the polarization tensor while $V$ multiplies the anti-symmetric Pauli matrix $\sigma^{(2)}$. This part describes a phase difference between $E_{1}$ and $E_{2}$ which results in circular polarization. This is best seen by expressing $P_{a b}$ in terms of the helicity basis $\boldsymbol{\varepsilon}^{( \pm)}=\frac{1}{\sqrt{2}}\left(\boldsymbol{\varepsilon}^{(1)} \pm i \boldsymbol{\varepsilon}^{(2)}\right)$, where one finds that $V$ is the difference between the left and right handed circular polarized intensities (see e.g. [25]). Thomson scattering does not introduce circular polarization. We therefore expect the $V$-Stokes parameter of the CMB radiation to vanish. We neglect it in the following. If $V=0$, we have $P_{a b}=P_{a b}^{*}=P_{b a}$. Hence $P_{a b}$ is a real, symmetric, traceless matrix.

We define also

$$
\begin{aligned}
P & \equiv P_{++}=2 P^{a b} \boldsymbol{\varepsilon}_{a}^{(+)} \boldsymbol{\varepsilon}_{b}^{(+)}=Q+i U \text { and } \\
\bar{P} & \equiv P_{--}=2 P^{a b} \overline{\boldsymbol{\varepsilon}}_{a}^{(+)} \overline{\boldsymbol{\varepsilon}}_{b}^{(+)}=2 P^{a b} \boldsymbol{\varepsilon}_{a}^{(-)} \boldsymbol{\varepsilon}_{b}^{(-)}=Q-i U .
\end{aligned}
$$

Rotating the basis $\left(\boldsymbol{\varepsilon}^{(1)}, \boldsymbol{\varepsilon}^{(2)}\right)$ by an angle $\phi$ around the direction $\mathbf{n}$, the polarization turns like

$$
P_{ \pm \pm} \rightarrow e^{ \pm 2 i \phi} P_{ \pm \pm}
$$

$P_{a b}$ is a spin 2 tensor field on the sphere and $P_{ \pm \pm}$are its helicity \pm 2 components. Such a tensor field can be expanded in terms of spin weighted spherical harmonics, ${ }_{s} Y_{\ell m}$. For $s=0$ these are the usual spherical harmonics and for $s \neq 0$ they depend not only on $\mathbf{n}$ but also on the basis $\left(\boldsymbol{\varepsilon}^{(1)}, \boldsymbol{\varepsilon}^{(2)}\right)$ on the sphere which is traditionally taken to be $\left(\mathbf{e}_{\vartheta}, \mathbf{e}_{\varphi}\right)$, where $\mathbf{e}_{\vartheta}=\partial_{\vartheta}$ and $\mathbf{e}_{\varphi}=\frac{1}{\sin \vartheta} \partial_{\varphi}$. The spin weighted spherical harmonics are eigenfunctions of the Laplace operator on the sphere with eigenvalue $-\ell(\ell+1)$ and they transform with helicity $s$ under rotations around $\mathbf{n}$. For $\ell<s$ they vanish, ${ }_{s} Y_{\ell m}=0$. More properties of the spin weighted spherical harmonics and especially their relation to the matrix elements of the irreducible representations of the rotation group can be found in [3].

The perturbations $\mathscr{M}$ is nothing else than the relative perturbation of the intensity, $\mathscr{M}=\frac{1}{4} \delta I / I$. Correspondingly we define the dimensionless perturbation variables

$$
\mathscr{Q}=\frac{Q}{4 I} \quad \text { and } \quad \mathscr{U}=\frac{U}{4 I}, \quad \mathscr{P}_{++}=\frac{P_{++}}{4 I}=\mathscr{Q}+i \mathscr{U}, \quad \mathscr{P}_{--}=\frac{P_{--}}{4 I}=\mathscr{Q}-i \mathscr{U} .
$$


Since the polarization of the background vanishes, these are both gauge-invariant. We can now expand the polarization in spin weighted spherical harmonics,

$$
\begin{aligned}
\mathscr{P}_{ \pm, \pm}=(\mathscr{Q} \pm i \mathscr{U})(\mathbf{n}) & =\sum_{\ell=2}^{\infty} \sum_{m=-\ell}^{\ell} a_{\ell m}^{( \pm 2)}{ }_{ \pm 2} Y_{\ell m}(\mathbf{n}) \\
& =\sum_{\ell=2}^{\infty} \sum_{m=-\ell}^{\ell}\left(e_{\ell m} \pm i b_{\ell m}\right){ }_{ \pm 2} Y_{\ell m}(\mathbf{n}) .
\end{aligned}
$$

Hence

$$
e_{\ell m}=\frac{1}{2}\left(a_{\ell m}^{(2)}+a_{\ell m}^{(-2)}\right), \quad b_{\ell m}=\frac{-i}{2}\left(a_{\ell m}^{(2)}-a_{\ell m}^{(-2)}\right) .
$$

Under a 'parity' transformation, $\mathbf{n} \rightarrow-\mathbf{n}$ the basis vectors $\mathbf{e}^{( \pm)}$transform as $\mathbf{e}^{( \pm)} \rightarrow \mathbf{e}^{(\mp)}$. Hence the coefficient $a_{\ell m}^{(2)}$ turns into $a_{\ell m}^{(-2)}$ and $a_{\ell m}^{(-2)} \rightarrow a_{\ell m}^{(2)}$ so that $e_{\ell m}$ remains invariant while $b_{\ell m}$ changes sign.

The expansion in $a_{\ell m}^{( \pm 2)}$ is a decomposition into positive and negative helicity, while $\left(e_{\ell m}, b_{\ell m}\right)$ is a decomposition into the $\mathscr{Q}$ and $\mathscr{U}$ Stokes parameter with respect to the canonical basis on the sphere which requires the choice of a $z$-axis.

One can also define differential operators which are spin raising and lowering operators $\not$ and $\partial^{*}$ (see [3]), similar to the quantum mechanical angular momentum operators $L_{+}$and $L_{-}$ which raise and lower the magnetic quantum number $m$. The operators $\partial^{(*)}$ have the properties $\partial_{s} Y_{\ell m} \propto{ }_{s+1} Y_{\ell m}$ and $\partial^{*}{ }_{s} Y_{\ell m} \propto{ }_{s-1} Y_{\ell m}$. Actually one obtains

$$
\begin{aligned}
\partial^{2}\left({ }_{2} Y_{\ell m}\right) & =\sqrt{\frac{(\ell+2) !}{(\ell-2) !}} Y_{\ell m} \\
\left(\partial^{*}\right)^{2}\left({ }_{2} Y_{\ell m}\right) & =\sqrt{\frac{(\ell+2) !}{(\ell-2) !}} Y_{\ell m} .
\end{aligned}
$$

Applying this to $\mathscr{Q} \pm i \mathscr{U}$ we find

$$
\begin{aligned}
\left(\partial^{*}\right)^{2}(\mathscr{Q}+i \mathscr{U})(\mathbf{n})=\tilde{\mathscr{E}}(\mathbf{n})+i \tilde{\mathscr{B}}(\mathbf{n}) & =\sum_{\ell=2}^{\infty} \sum_{m=-\ell}^{\ell} a_{\ell m}^{(2)} \sqrt{\frac{(\ell+2) !}{(\ell-2) !}} Y_{\ell m}(\mathbf{n}), \\
\partial^{2}(\mathscr{Q}-i \mathscr{U})(\mathbf{n})=\tilde{\mathscr{E}}(\mathbf{n})-i \tilde{\mathscr{B}}(\mathbf{n}) & =\sum_{\ell=2}^{\infty} \sum_{m=-\ell}^{\ell} a_{\ell m}^{(-2)} \sqrt{\frac{(\ell+2) !}{(\ell-2) !}} Y_{\ell m}(\mathbf{n}), \\
\text { where } \tilde{\mathscr{E}}(\mathbf{n}) & =\sum_{\ell=2}^{\infty} \sum_{m=-\ell}^{\ell} e_{\ell m} \sqrt{\frac{(\ell+2) !}{(\ell-2) !}} Y_{\ell m}(\mathbf{n}), \\
\tilde{\mathscr{B}}(\mathbf{n}) & =\sum_{\ell=2}^{\infty} \sum_{m=-\ell}^{\ell} b_{\ell m} \sqrt{\frac{(\ell+2) !}{(\ell-2) !}} Y_{\ell m}(\mathbf{n}) .
\end{aligned}
$$

We also define the (non-local) scalar quantities

$$
\begin{aligned}
& \mathscr{E}(\mathbf{n})=\sum_{\ell=2}^{\infty} \sum_{m=-\ell}^{\ell} e_{\ell m} Y_{\ell m}(\mathbf{n}), \\
& \mathscr{B}(\mathbf{n})=\sum_{\ell=2}^{\infty} \sum_{m=-\ell}^{\ell} b_{\ell m} Y_{\ell m}(\mathbf{n}) .
\end{aligned}
$$


Like temperature fluctuations, $\mathscr{E}$ and $\mathscr{B}$ are invariant under rotation. Since the sign of $b_{\ell m}$ changes under parity, $\mathscr{B}$ has negative parity while $\mathscr{E}$ and $\mathscr{M}$ have positive parity. Actually one can show [3] that $\left(\not^{*}\right)^{2}=2\left(\nabla_{-}\right)^{2}$ and $(\not \partial)^{2}=2\left(\nabla_{+}\right)^{2}$, so that

$$
\begin{aligned}
\tilde{\mathscr{E}} & =\nabla_{-} \nabla_{-} \mathscr{P}_{++}+\nabla_{+} \nabla_{+} \mathscr{P}_{--}=2 \nabla_{i} \nabla_{j} \mathscr{P}_{i j}=2 \operatorname{div} \operatorname{div} \mathscr{P}, \\
\tilde{\mathscr{B}} & =\nabla_{-} \nabla_{-} \mathscr{P}_{++}-\nabla_{+} \nabla_{+} \mathscr{P}_{--}=2 \varepsilon_{l m} \varepsilon_{i j} \nabla_{l} \nabla_{i} \mathscr{P}_{j m}=2 \operatorname{rot} \operatorname{rot} \mathscr{P} .
\end{aligned}
$$

Hence $\mathscr{E}$ measures gradient contributions while $\mathscr{B}$ measures curl contributions to the electric field considered as a function on the sphere as shown in Fig. 3. (The electric field is transverse and hence tangent to the sphere of photon directions.)
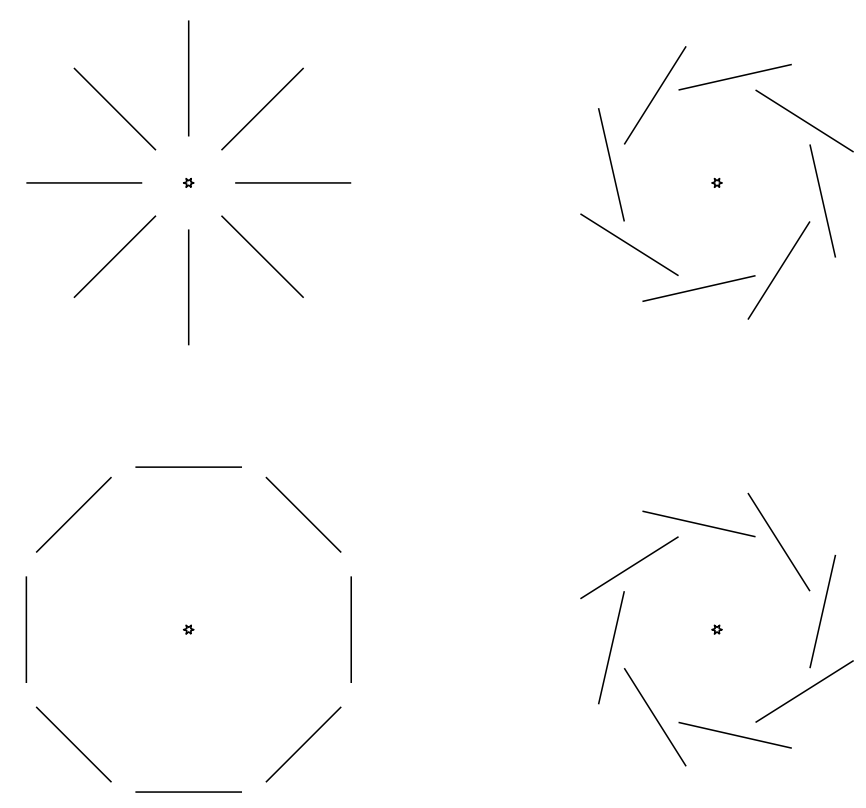

Figure 3: $E$-polarization (left) and $B$-polarization (right) patterns are shown around the photon direction indicated as the central asterisk. $E$ polarization can be either radial or tangential, while $B$ polarization is clearly of curl type.

\subsection{The collision term}

So far, we have not discussed Thomson scattering which is the relevant scattering process right before recombination. To study it we consider an incoming photon from direction $\mathbf{n}^{\prime}$ which is then scattered into direction $\mathbf{n}$ with $\mathbf{n} \cdot \mathbf{n}^{\prime}=\cos \beta$. For photons which are polarized in the scattering plane, the scattered field amplitude is suppressed by a factor $|\cos \beta|$, while normal to the plane it is not suppressed. In the rest frame of the electron one finds, see [25]

$$
\begin{aligned}
& E_{\|}^{(c)}=\frac{n_{e} e^{2}}{m_{e}} \cos \beta E_{\|}=\sqrt{\frac{3}{8 \pi} n_{e} \sigma_{T}} \cos \beta E_{\|} \\
& E_{\perp}^{(c)}=\frac{n_{e} e^{2}}{m_{e}} E_{\perp}=\sqrt{\frac{3}{8 \pi} n_{e} \sigma_{T}} E_{\perp} .
\end{aligned}
$$


Defining the vector

$$
\mathscr{V}=\left(\begin{array}{c}
\mathscr{M} \\
\mathscr{Q}+i \mathscr{U} \\
\mathscr{Q}-i \mathscr{U}
\end{array}\right)
$$

we can derive the following form of the collision term in the background frame [3] (see Chandrasekhar for the original derivation [26])

$$
\begin{aligned}
C[\mathscr{V}]=a n_{e} \sigma_{T}\left[\frac{1}{10} \int \Omega_{\mathbf{n}^{\prime}} \sum_{m=-2}^{2} P_{m}\left(\mathbf{n}, \mathbf{n}^{\prime}\right) \mathscr{V}\left(\mathbf{n}^{\prime}\right)-\mathscr{V}(\mathbf{n})\right. \\
\left.+\left[\frac{1}{4 \pi} \int \Omega_{\mathbf{n}^{\prime}} \mathscr{M}\left(\mathbf{n}^{\prime}\right)+\mathbf{n} \cdot \mathbf{V}^{(b)}\right]\left(\begin{array}{l}
1 \\
0 \\
0
\end{array}\right)\right]
\end{aligned}
$$

where $P_{m}\left(\mathbf{n}, \mathbf{n}^{\prime}\right)$ is given in terms of spin weighted spherical harmonics and $m=0$ couples to scalar perturbations, $m= \pm 1$ to vector perturbations and $m= \pm 2$ to tensor perturbations

$$
P_{m}\left(\mathbf{n}, \mathbf{n}^{\prime}\right)=\left(\begin{array}{ccc}
Y_{2 m}(\mathbf{n}) Y_{2 m}^{*}\left(\mathbf{n}^{\prime}\right) & -\sqrt{\frac{3}{2}} Y_{2 m}(\mathbf{n}){ }_{2} Y_{2 m}^{*}\left(\mathbf{n}^{\prime}\right) & -\sqrt{\frac{3}{2}} Y_{2 m}(\mathbf{n}){ }_{2} Y_{2 m}^{*}\left(\mathbf{n}^{\prime}\right) \\
-\sqrt{6}{ }_{2} Y_{2 m}(\mathbf{n}) Y_{2 m}^{*}\left(\mathbf{n}^{\prime}\right) & 3{ }_{2} Y_{2 m}(\mathbf{n})_{2} Y_{2 m}^{*}\left(\mathbf{n}^{\prime}\right) & 3{ }_{2} Y_{2 m}(\mathbf{n}){ }_{2} Y_{2 m}^{*}\left(\mathbf{n}^{\prime}\right) \\
-\sqrt{6}{ }_{-2} Y_{2 m}(\mathbf{n}) Y_{2 m}^{*}\left(\mathbf{n}^{\prime}\right) & 3{ }_{-2} Y_{2 m}(\mathbf{n})_{2} Y_{2 m}^{*}\left(\mathbf{n}^{\prime}\right) & 3{ }_{-2} Y_{2 m}(\mathbf{n})_{-2} Y_{2 m}^{*}\left(\mathbf{n}^{\prime}\right)
\end{array}\right) .
$$

The Boltzmann equation can then be written for the Fourier modes. This is best done using the total angular momentum method (see [27,3]) from which finally the CMB power spectra can be determined as integrals over wave numbers,

$$
\begin{gathered}
C_{\ell}^{(\mathscr{M})}=\left\langle\left|a_{\ell m}\right|^{2}\right\rangle \quad \text { where } \\
\mathscr{M}(\mathbf{x}, \mathbf{n})=\sum_{\ell=0}^{\infty} \sum_{m=-\ell}^{\ell} a_{\ell m}(x) Y_{\ell m}(\mathbf{n}) \\
C_{\ell}^{(\mathscr{E})}=\left\langle\left|e_{\ell m}\right|^{2}\right\rangle \\
C_{\ell}^{(\mathscr{B})}=\left\langle\left|b_{\ell m}\right|^{2}\right\rangle \\
C_{\ell}^{(\mathscr{M} \mathscr{E})}=\left\langle a_{\ell m}^{*} e_{\ell m}\right\rangle .
\end{gathered}
$$

These spectra can be calculated very rapidly using one of the publicly available codes, CMBfast [28], the original or CAMBCODE [29] the presently most powerful code or CMBEASY [30], the most user friendly code. More details can be found in [3].

\section{Observations and parameter estimation}

Since the perturbation equations are linear, the CMB anisotropy and polarization amplitudes depend linearly on the initial conditions. Let us, for simplicity (and also since this is the situation for most inflationary models), assume that the initial conditions are given by two power spectra,

$$
\begin{aligned}
(2 \pi)^{3} P_{\Psi}(k) \delta\left(\mathbf{k}-\mathbf{k}^{\prime}\right) & =\left\langle\Psi\left(\mathbf{k}, t_{\text {in }}\right) \Psi^{*}\left(\mathbf{k}^{\prime}, t_{\text {in }}\right)\right\rangle \quad \text { and } \\
(2 \pi)^{3} P_{h}(k) \delta\left(\mathbf{k}-\mathbf{k}^{\prime}\right) & =\left\langle H_{+2}\left(\mathbf{k}, t_{\text {in }}\right) H_{+2}^{*}\left(\mathbf{k}^{\prime}, t_{\text {in }}\right)\right\rangle=\left\langle H_{-2}\left(\mathbf{k}, t_{\text {in }}\right) H_{-2}^{*}\left(\mathbf{k}^{\prime}, t_{\text {in }}\right)\right\rangle .
\end{aligned}
$$



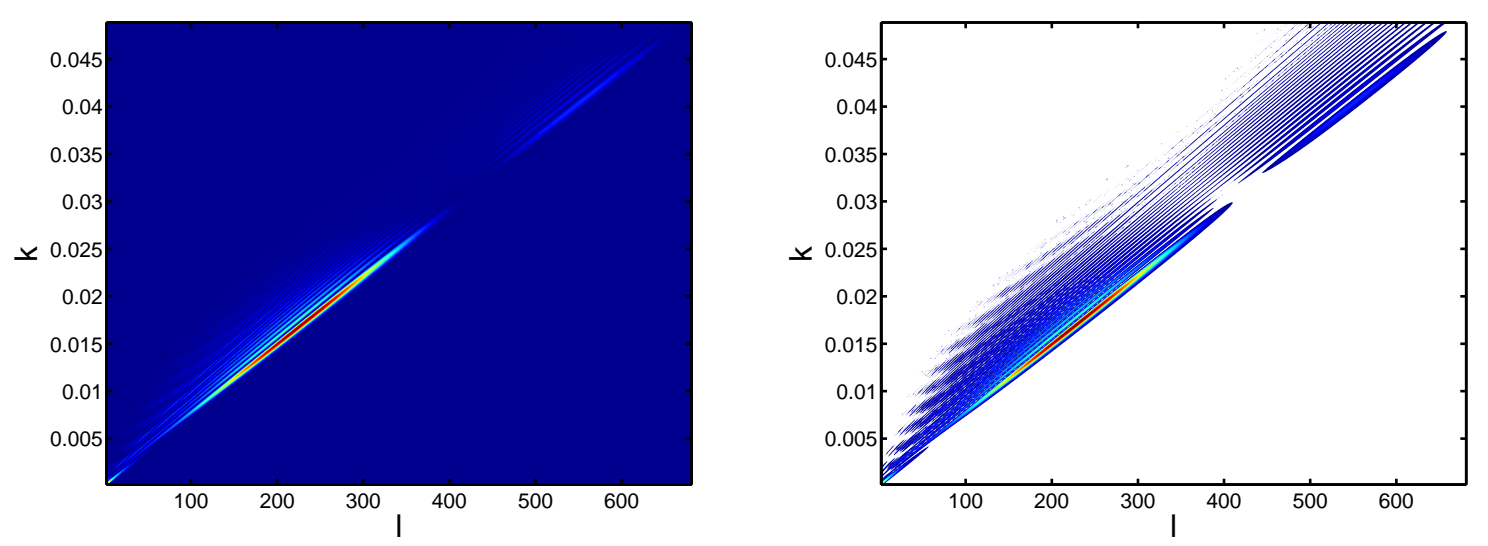

Figure 4: The scalar CMB anisotropy transfer function $T_{\ell}^{(T)}(k, 0)$ is shown. The first two acoustic peaks and the SW plateau are clearly visible. In the presentation on the left hand panel all points with amplitude smaller that $\frac{1}{40}$ of the maximum are set to dark blue while on the right hand panel they are set to white. It is interesting to see how narrow the $(k, \ell)$ correlation is. In the right hand panel the 'ringing' due to secondary maxima of the Bessel functions is better visible. The cosmological parameters chosen for this plot are $\Omega_{\Lambda}=0.73, \Omega_{m} h^{2}=0.13, \Omega_{b} h^{2}=0.022$ and $K=0$.

As the final perturbations depend linearly on the initial conditions, we can express the amplitudes $a_{\ell m}, e_{\ell m}$ and $b_{\ell m}$ in the form

$$
\begin{aligned}
a_{\ell m} & =\int d^{3} k T_{\ell m}^{(T)}(\mathbf{k}, 0) \Psi\left(\mathbf{k}, t_{\mathrm{in}}\right)+T_{\ell m}^{(T)}(\mathbf{k},+2) H_{+2}\left(\mathbf{k}, t_{\mathrm{in}}\right)+T_{\ell m}^{(T)}(\mathbf{k},-2) H_{-2}\left(\mathbf{k}, t_{\mathrm{in}}\right), \\
e_{\ell m} & =\int d^{3} k T_{\ell m}^{(E)}(\mathbf{k}, 0) \Psi\left(\mathbf{k}, t_{\mathrm{in}}\right)+T_{\ell m}^{(E)}(\mathbf{k},+2) H_{+2}\left(\mathbf{k}, t_{\mathrm{in}}\right)+T_{\ell m}^{(E)}(\mathbf{k},-2) H_{-2}\left(\mathbf{k}, t_{\mathrm{in}}\right), \\
b_{\ell m} & =\int d^{3} k T_{\ell m}^{(B)}(\mathbf{k},+2) H_{+2}\left(\mathbf{k}, t_{\mathrm{in}}\right)+T_{\ell m}^{(B)}(\mathbf{k},-2) H_{-2}\left(\mathbf{k}, t_{\mathrm{in}}\right),
\end{aligned}
$$

for some 'transfer functions' $T_{\ell m}^{(X)}$. For the power spectra we then obtain

$$
\begin{aligned}
C_{\ell}^{(T)} & =4 \pi \int \frac{d k}{k}\left[\left|T_{\ell}^{(T)}(k, 0)\right|^{2} k^{3} P_{\Psi}(k)+2\left|T_{\ell}^{(T)}(k, 2)\right|^{2} k^{3} P_{h}(k)\right], \\
C_{\ell}^{(T E)} & =4 \pi \int \frac{d k}{k}\left[T_{\ell}^{(T E)}(k, 0) k^{3} P_{\Psi}(k)+2 T_{\ell}^{(T E)}(k, 2) k^{3} P_{h}(k)\right], \\
C_{\ell}^{(E)} & =4 \pi \int \frac{d k}{k}\left[\left|T_{\ell}^{(E)}(k, 0)\right|^{2} k^{3} P_{\Psi}(k)+2\left|T_{\ell}^{(E)}(k, 2)\right|^{2} k^{3} P_{h}(k)\right], \\
C_{\ell}^{(B)} & =8 \pi \int \frac{d k}{k}\left|T_{\ell}^{(T)}(k, 2)\right|^{2} k^{3} P_{h}(k),
\end{aligned}
$$

where we have defined the direction integrated transfer functions,

$$
\begin{aligned}
\left|T_{\ell}^{(X)}(k, s)\right|^{2} & =\frac{1}{4 \pi} \int d \Omega_{\hat{\mathbf{k}}}\left|T_{\ell m}^{(X)}(\mathbf{k}, s)\right|^{2}, \\
T_{\ell}^{(T E)}(k, s) & =\frac{1}{4 \pi} \int d \Omega_{\hat{\mathbf{k}}} T_{\ell m}^{(T)}(\mathbf{k}, s) T_{\ell m}^{(E) *}(\mathbf{k}, s) .
\end{aligned}
$$

Because of statistical isotropy, these integrals do not depend on $m$. Note that $T_{\ell}^{(T E)}(k, s)$ can be negative since both $T_{\ell m}^{(T)}(\mathbf{k}, s)$ and $T_{\ell m}^{(E) *}(\mathbf{k}, s)$ are in general complex Fourier transforms of real 
functions, so that $\left(T_{\ell m}^{(T)}(\mathbf{k}, s) T_{\ell m}^{(E) *}(\mathbf{k}, s)\right)^{*}=T_{\ell m}^{(T)}(-\mathbf{k}, s) T_{\ell m}^{(E) *}(-\mathbf{k}, s)$. Therefore the integral (4.6) is real but not necessarily positive. We have also used that scalar and tensor perturbations are uncorrelated. These transfer functions only depend on the background cosmology, hence on cosmological parameters. However, as is clear from Eqs. (4.1) to (4.4), we need to know the initial power spectra $P_{\Psi}$ and $P_{h}$ to infer the transfer functions and hence the cosmological parameters from the observed power spectra. Usually this is done by parameterizing the initial conditions with a few parameters, e.g., $P_{\Psi}(k) k^{3}=A_{S}\left(k t_{0}\right)^{n_{S}-1}$ and $P_{h}(k) k^{3}=A_{T}\left(k t_{0}\right)^{n_{T}}$. One then fits jointly the parameters describing the initial conditions and the cosmological parameters which determine the transfer functions.

The presently available data on temperature anisotropy and on polarization are shown in Figs. 5, 6 and 7
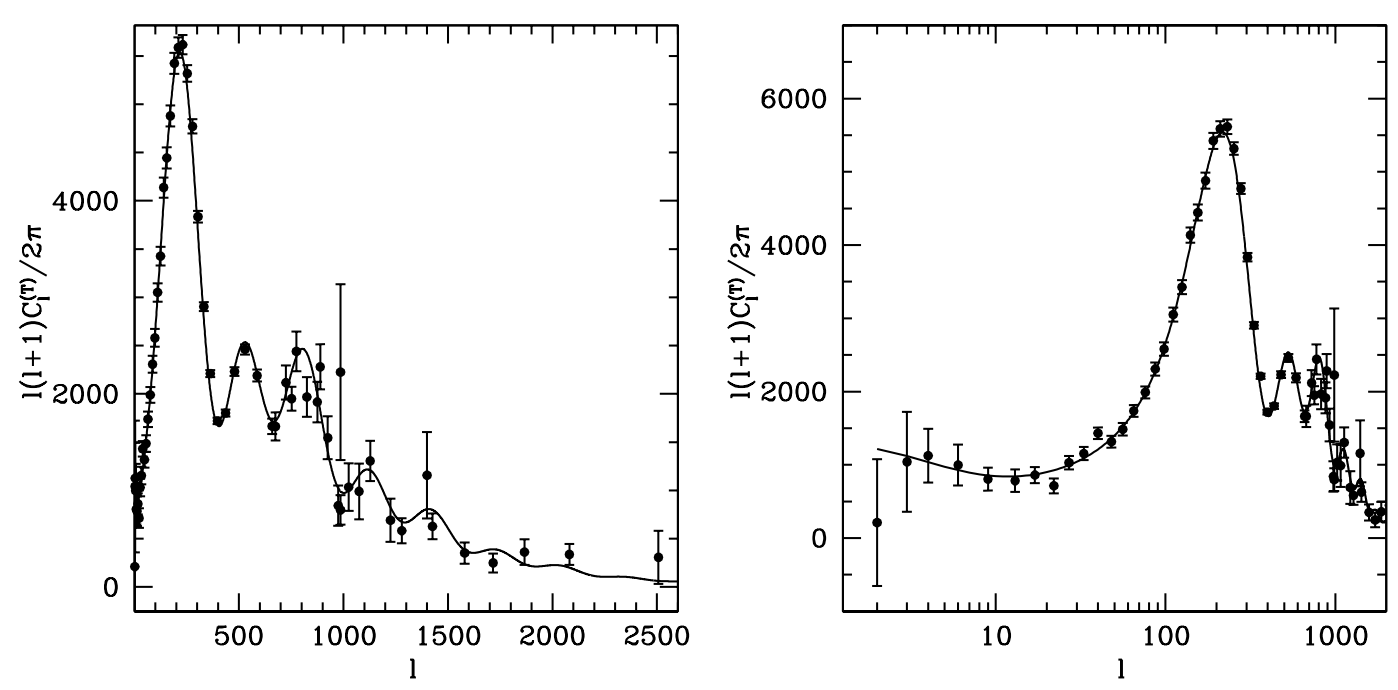

Figure 5: The observed CMB anisotropy spectrum from WMAP [31] extended by Boomerang [32] and Acbar [33] in linear (left) and log (right) scale. The line draws the best fit $\Lambda$ CDM model.

Also shown in these figures is a line which is the best fit $\Lambda$ CDM model. Obtained by a Markov Chain Monte Carlo (MCMC) method [35] parameter fit to the data. More details about parameter fitting can be found in [3]. Here we just want to present the best presently available cosmological parameters from different data sets and obtained making different assumptions.

\subsection{Degeneracies}

When estimating cosmological parameters with an MCMC method, the resulting best fit parameters and the error bars depend on the model assumptions. Therefore, we must be very careful when interpreting the results. Clearly, when allowing for more model parameters, the errors do in general increase. But there is a much more serious problem: degeneracies. The transfer functions depend strongly on certain combinations of cosmological parameters like the angular diameter distance to the last scattering surface, which determines the positions of the acoustic peaks and $\Omega_{m} h^{2}$ which determines the amplitude of the gravitational potential at the last scattering surface, as well 


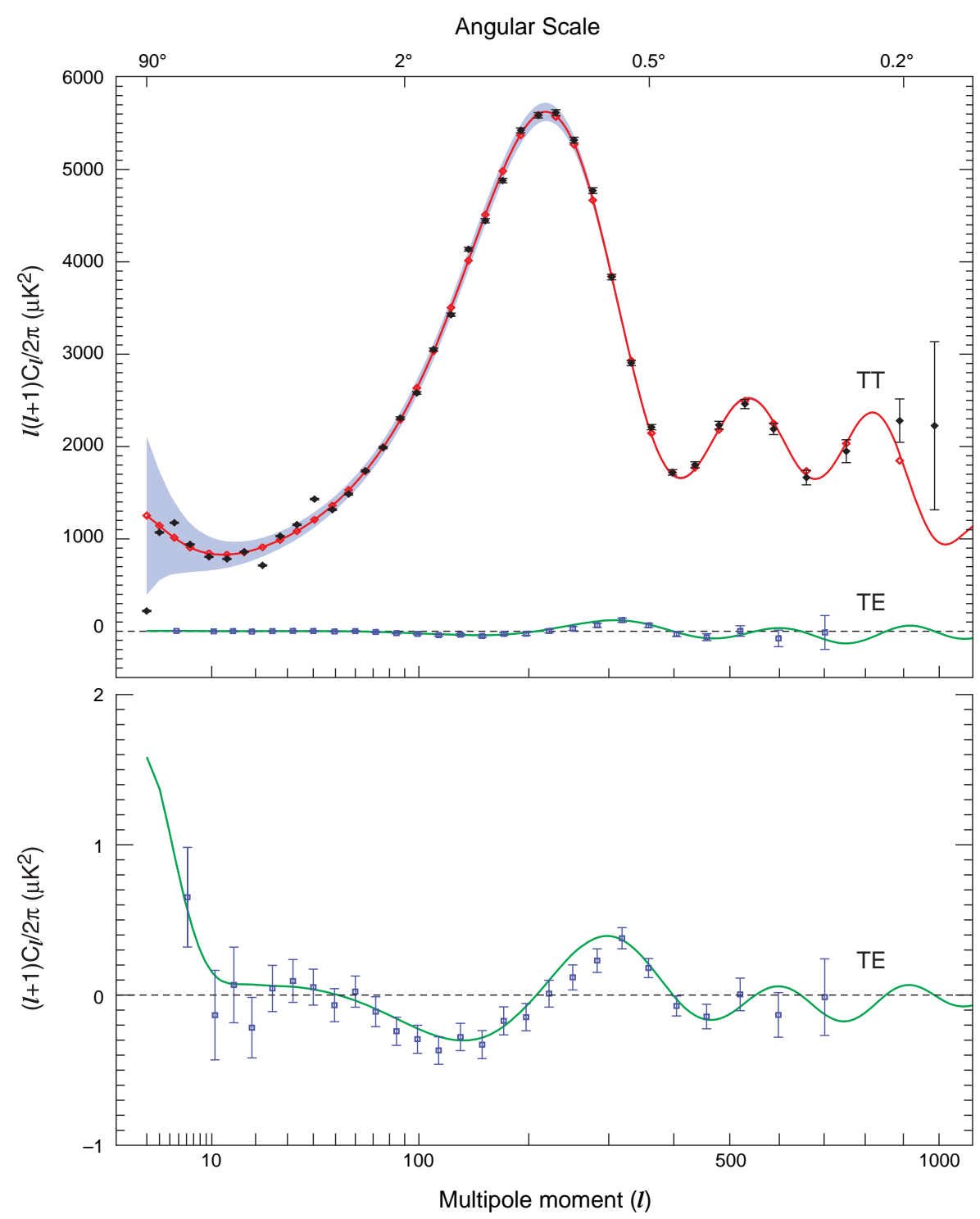

Figure 6: The CMB anisotropy spectrum and the temperature-polarization cross-correlation obtained from the WMAP 3 year data (figure from [31]).

as $\Omega_{b} h^{2}$ which determines the asymmetry of even and odd peaks and the damping scale. However, when keeping $\left(d_{A}, \Omega_{m} h^{2}, \Omega_{b} h^{2}\right)$ fixed and varying e.g., curvature and the Hubble parameter, the CMB anisotropy and polarization spectra remain virtually unchanged, see Fig. 8.

To lift such degeneracies we usually need to resort to complementary data, like the Hubble key project measurement of the Hubble parameter, $h=0.72 \pm 0.08$ or galaxy surveys which are sensitive to the combination $\Omega_{m} h$ etc. The most important lesson is of course that we do need as much complementary data as possible, since first of all we need to lift the degeneracies in the parameter dependence of the $\mathrm{CMB}$ and secondly the values of cosmological parameters determined by MCMC methods are always more or less model dependent. 


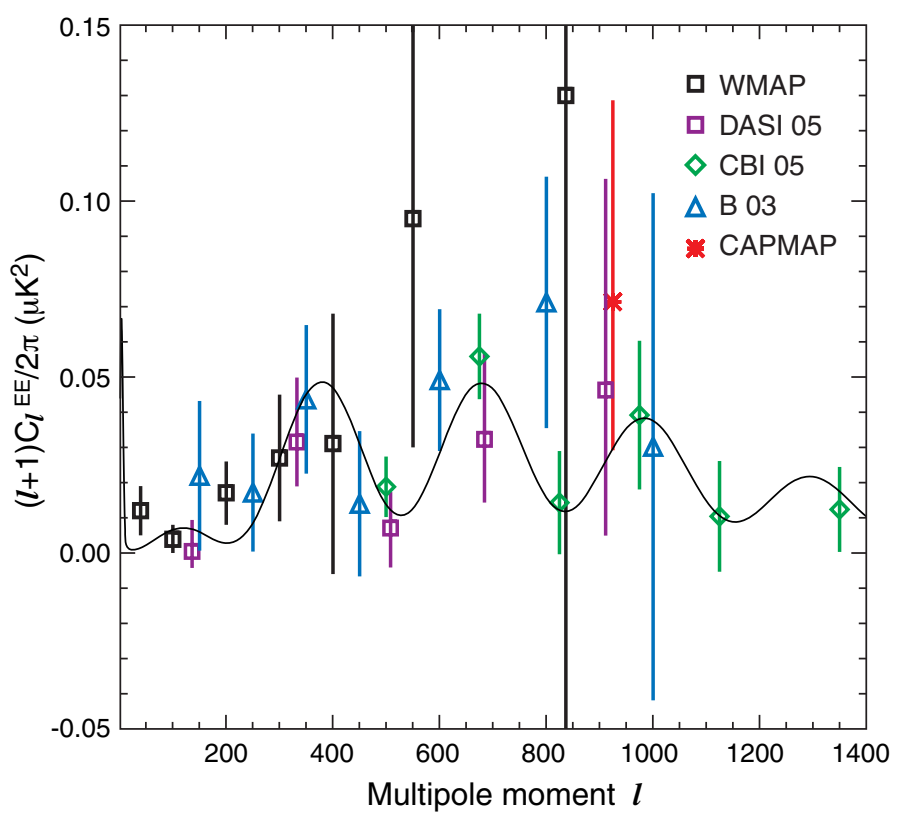

Figure 7: The measured EE polarization spectrum from Wmap 3 year, Boomerang and others. For more details see [34] from where this figure is taken.

\begin{tabular}{|c|c|c|c|c|}
\hline Parameter & $\begin{array}{l}\text { WMAP } \\
\text { Only }\end{array}$ & $\begin{array}{c}\text { WMAP } \\
+\mathrm{CBI}+\mathrm{VSA}\end{array}$ & $\begin{array}{l}\text { WMAP+ACBAR } \\
\text { +BOOMERanG }\end{array}$ & $\begin{array}{l}\text { WMAP + } \\
\text { 2dFGRS }\end{array}$ \\
\hline$\overline{100 \Omega_{b} h^{2}}$ & $2.233_{-0.091}^{+0.072}$ & $2.212_{-0.084}^{+0.066}$ & $2.231_{-0.088}^{+0.070}$ & $2.223_{-0.083}^{+0.066}$ \\
\hline$\Omega_{m} h^{2}$ & $0.1268_{-0.0095}^{+0.0072}$ & $0.1233_{-0.0086}^{+0.0070}$ & $0.1259_{-0.0095}^{+0.0077}$ & $0.1262_{-0.0062}^{+0.0045}$ \\
\hline$h$ & $0.734_{-0.038}^{+0.028}$ & $0.743_{-0.037}^{+0.0270}$ & $0.739_{-0.038}^{+0.028}$ & $0.732_{-0.025}^{+0.018}$ \\
\hline$A$ & $0.801_{-0.054}^{+0.043}$ & $0.796_{-0.052}^{+0.041}$ & $0.798_{-0.054}^{+0.038}$ & $0.799_{-0.051}^{-0.023}$ \\
\hline$\tau$ & $0.088_{-0.034}^{+0.028}$ & $0.088_{-0.033}^{+0.027}$ & $0.088_{-0.033}^{+0.030}$ & $0.083_{-0.031}^{+0.027}$ \\
\hline$n_{s}$ & $0.951_{-0.019}^{+0.015}$ & $0.947_{-0.017}^{+0.0013}$ & $0.951_{-0.020}^{+0.033}$ & $0.948_{-0.018}^{+0.014}$ \\
\hline$\overline{\sigma_{8}}$ & $0.744_{-0.060}^{+0.050}$ & $0.722_{-0.053}^{+0.043}$ & $0.739_{-0.059}^{+0.047}$ & $0.737_{-0.045}^{+0.033}$ \\
\hline$\Omega_{m}$ & $0.238_{-0.041}^{+0.030}$ & $0.226_{-0.036}^{+0.026}$ & $0.233_{-0.041}^{+0.029}$ & $0.236_{-0.024}^{+0.016}$ \\
\hline
\end{tabular}

Table 1: Joint Likelihoods for a flat $\Lambda$ CDM model with purely scalar perturbations. The WMAP three year data are combined with small scale CMB experiments (CBI+VSA, BOOMERanG) or galaxy survey data (2dFGRS). $A$ is the amplitude of density fluctuations at $k=0.002 / \mathrm{Mpc}$ and $\tau$ is the optical depth to the last scattering surface. The parameters $\sigma_{8}$ and $\Omega_{m}$ are derived. Table from [36].

\section{Conclusions}

In these lectures we have explained to you that one of the main importance of the CMB is that it can nearly fully be calculated within linear perturbation theory and therefore these calculations can be performed relatively easily to high accuracy. In cosmology high accuracy means 0.5 to $1 \%$. Doing better than this is difficult and is a subject of present research. We have given an 


\begin{tabular}{|c||c|c|c|c|}
\hline \hline & WMAP+ & WMAP+ & WMAP + & WMAP+ \\
& SDSS & SNLS & SN Gold & CFHTLS \\
Parameter & & & & \\
\hline \hline $100 \Omega_{b} h^{2}$ & $2.233_{-0.086}^{+0.062}$ & $2.233_{-0.088}^{+0.069}$ & $2.227_{-0.082}^{+0.065}$ & $2.255_{-0.083}^{+0.062}$ \\
$\Omega_{m} h^{2}$ & $0.1329_{-0.0075}^{+0.0056}$ & $0.1295_{-0.0072}^{+0.0056}$ & $0.1349_{-0.0071}^{+0.0056}$ & $0.1408_{-0.0050}^{+0.0034}$ \\
$h$ & $0.709_{-0.032}^{+0.024}$ & $0.723_{-0.030}^{+0.021}$ & $0.701_{-0.026}^{+0.020}$ & $0.687_{-0.024}^{+0.016}$ \\
$A$ & $0.813_{-0.052}^{+0.042}$ & $0.808_{-0.051}^{+0.044}$ & $0.827_{-0.045}^{+0.053}$ & $0.846_{-0.047}^{+0.037}$ \\
$\tau$ & $0.079_{-0.032}^{+0.029}$ & $0.085_{-0.032}^{+0.028}$ & $0.079_{-0.034}^{+0.028}$ & $0.088_{-0.032}^{+0.026}$ \\
$n_{s}$ & $0.948_{-0.018}^{+0.015}$ & $0.950_{-0.019}^{+0.015}$ & $0.946_{-0.019}^{+0.015}$ & $0.953_{-0.019}^{+0.015}$ \\
\hline \hline$\sigma_{8}$ & $0.772_{-0.048}^{+0.036}$ & $0.758_{-0.052}^{+0.038}$ & $0.784_{-0.049}^{+0.035}$ & $0.826_{-0.035}^{+0.022}$ \\
$\Omega_{m}$ & $0.266_{-0.036}^{+0.026}$ & $0.249_{-0.031}^{+0.024}$ & $0.276_{-0.031}^{+0.023}$ & $0.299_{-0.025}^{+0.019}$ \\
\hline
\end{tabular}

Table 2: As in Table 1 but including other data sets: galaxy surveys (SDSS), supernovae (SNLS and SN Gold) and weak lensing (CFHTLS). Table from [36].

overview of how CMB anisotropies and polarization are calculated and briefly discussed the main physical effects which enter. Then you have learned how, under simple assumptions for the initial conditions, these results can be used to estimate cosmological parameters.

Other interesting aspects of CMB anisotropies and polarizations which we have not touched upon in these lectures are, e.g., nonlinearities like CMB lensing [3] or non-Gaussianities. NonGaussianities are a very active field of research which contains a lot of additional information about inflation and about the non-linearities in the CMB. The problem is that most theories of inflation predict very small non-Gaussianities while most experimental setups will lead to non-Gaussian errors at some level. Therefore, the measurement of primordial non-Gaussianities so far remains a challenge for the future. A theoretical overview can be found e.g., in Ref. [37].

\section{Acknowledgment}

I thank the organizers for inviting me to teach in such a beautiful, inspiring place and the students for the many lively and stimulating discussions. I am very greatful to Martin Kunz for his help with Figure 4, and to Camille Bonvin for carefully reading a first draft of this text. 

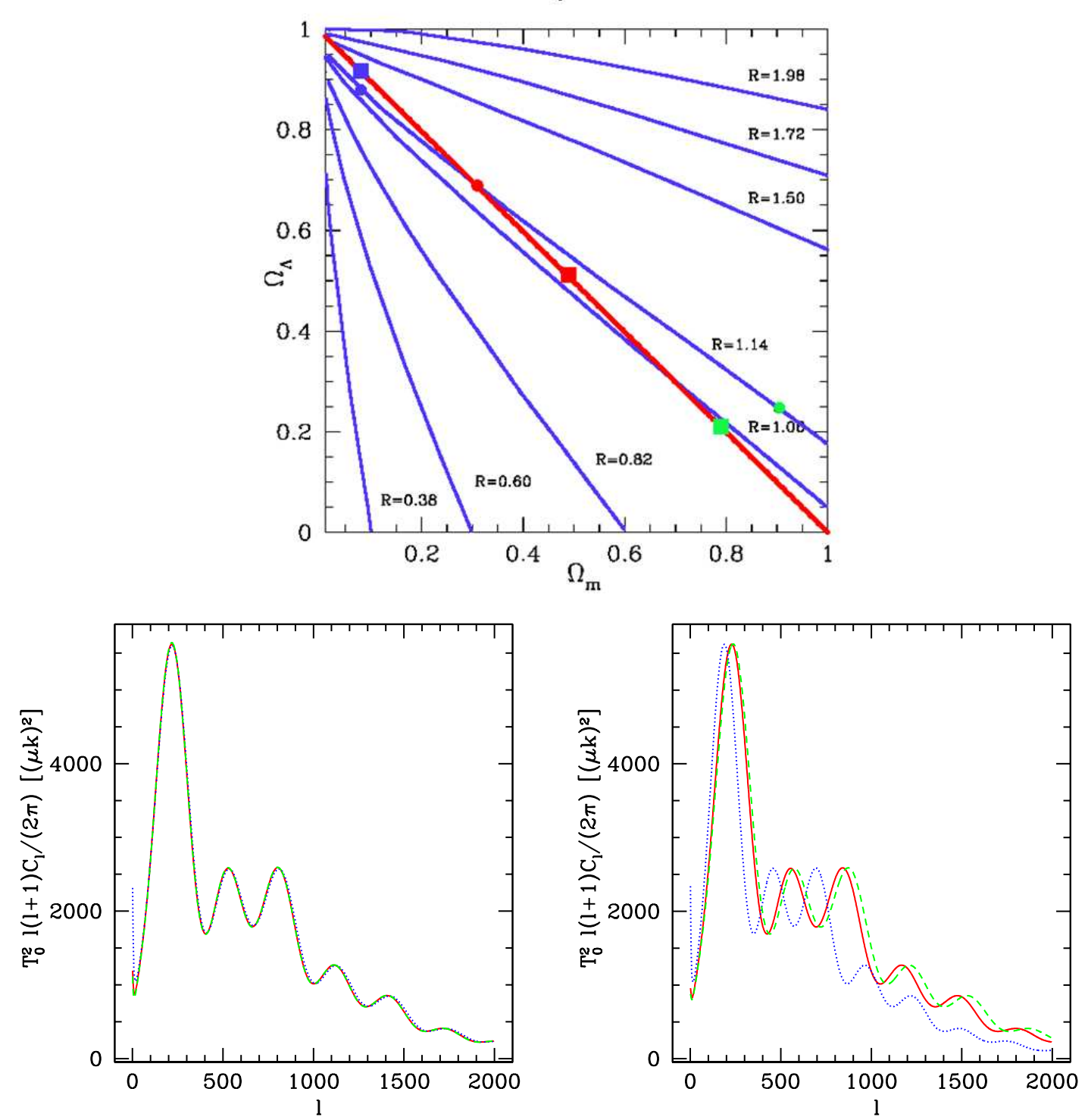

Figure 8: On the top panel lines of equal angular diameter distance are indicated. The number $R$ is the ratio of the angular diameter distance of the model to the one of a concordance model with $\Omega_{\Lambda}=0.7, \Omega_{m}=0.3$, $h=0.7$. The lines of constant curvature are parallel to the diagonal which is also drawn. In the lower left panel we show CMB anisotropy spectra with $\Omega_{K}>0$ (dashed), $\Omega_{K}<0$ (dotted) and $\Omega_{K}=0$ (solid), which have identical angular diameter distance, matter density and baryon density. They correspond to the bullets indicated in the top panel. The spectra overlay so precisely that we cannot distinguish them by eye. On the lower right panel we show three spectra with curvature zero, identical matter density and baryon density, but different angular diameter distances (the squares indicated in the top panel on the $K=0$ line). The spectra are significantly different. 


\section{References}

[1] A.A. Penzias and R.W. Wilson, A measurement of excess antenna temperature at $4080 \mathrm{Mc} / \mathrm{s}$, Astrophys. J. 142, 419 (1965).

[2] G.F. Smoot et al., Structure in the COBE differential microwave radiometer first-year maps Astrophys. J. 396, L1 (1992).

[3] R. Durrer, The Cosmic Microwave Background, Cambridge University Press (2008).

[4] J.M. Stewart and M. Walker, Perturbations of space-times in general relativity, Proc. R. Soc. London A341, 49 (1974).

[5] J. Bardeen, Gauge-invariant cosmological perturbations, Phys. Rev. D22, 1882 (1980).

[6] H. Kodama and M. Sasaki, Cosmological Perturbation Theory, Prog. Theor. Phys. Suppl 78, 1 (1984).

[7] V.F. Mukhanov, H.A. Feldman, and R.H. Brandenberger, Theory of Cosmological Perturbations, Phys. Rept. 215, 203 (1992).

[8] R. Durrer, Gauge invariant cosmological perturbation theory, Fund. of Cosmic Physics 15, 209 (1994).

[9] R.M. Wald, General Relativity, The University of Chicago Press 1984.

[10] G.F.R Ellis and M. Bruni, Covariant and gauge-invariant approach to cosmological density fluctuations, Phys. Rev. D40, 1804 (1989).

M. Bruni, P. Dunsby and G. Ellis, G. Cosmological perturbations and the physical meaning of gauge-invariant variables, Astrophys. J. 395, 34 (1992).

[11] R. Durrer and N. Straumann, Some Applications of the $3+1$ Formalism of General Relativity, Helv. Phys. Acta 61, 1027 (1988).

[12] G.B. Arfken and H.J. Weber, Mathematical Methods for Physicists, 5th edition, Academic Press (Hardcourt 2001).

[13] E. Lifshitz, About gravitational stability of expanding worlds, JETP 10, 116 (1946).

[14] R. Durrer, Gauge-invariant cosmological perturbation theory with seeds, Phys. Rev. D42, 2533 (1990).

[15] A. Kogut et al., Dipole Anisotropy in the CODE Differential Microwave Radiometers First-Year Sky Maps, Astrophys. J. 419, 1 (1993).

[16] R.K. Sachs and A.M. Wolfe, Perturbations of a Cosmological Model and Angular Variations of the Microwave Background, Astrophys. J. 147, 73 (1967).

[17] R. Durrer, M. Kunz and A. Melchiorri, Cosmic Structure Formation with Topological Defetcs, Phys. Rept. 364, 1 (2002).

[18] M. Tegmark et al., The 3D power spectrum of galaxies from the SDSS, Astrophys. J. 606, 702 (2004).

[19] P.J.E. Peebles, Principles of Physical Cosmology (Princeton University Press 1993).

[20] G.F. Smoot et al., Structure in the COBE differential microwave radiometer first-year maps Astrophys. J. 396, L1 (1992).

[21] C.L. Bennett et al. First-year Wilkinson Microwave Anisotropy Probe (WMAP) observations: Preliminary maps and basic results. Astrophys. J. Suppl. 148, 1 (2003). 
[22] R. Durrer, et al., Seeds of large-scale anisotropy in string cosmology, Phys. Rev. D 59, 043511 (1999).

[23] M. Bucher, K. Moodley and N. Turok, Constraining Iso-Curvature Perturbations with CMB Polarization, Phys. Rev. Lett. 87, 191301 (2001).

[24] R. Trotta, A. Riazuelo and R. Durrer Reproducing Cosmic Microwave Background anisotropies with mixed iso-curvature Perturbations, Phys. Rev. Lett., 87, 231301 (2001) and The cosmological constant and general iso-curvature initial conditions, Phys. Rev. D67, 063520 (2003).

[25] J.D. Jackson, Classical Electrodynamics, 2nd Edition, Wiley \& Sons (New Jork 1975).

[26] S. Chandrasekhar, Radiative Transfer, Dover (New York 1960).

[27] W. Hu and M. White, CMB Anisotropies: Total Angular Momenum Method, Phys. Rev. D56, 596 (1997).

[28] U. Seljak and M. Zaldarriaga, cfa-www.harvard.edu/ mzaldarr/CMBFAST/cmbfast.html .

[29] A. Lewis and A. Challinor, http: / / camb.info/

[30] M. Doran, www . cmbeasy . org/

[31] G. Hinshaw et al., Three-Year Wilkinson Microwave Anisotropy Probe (WMAP) Observations: Temperature Analysis, Astrophys. J. Suppl. 170, 288 (2007).

[32] W.C. Jones et al., A Measurement of the Angular Power Spectrum of the CMB Temperature Anisotropy from the 2003 Flight of Boomerang, Astrophys. J. 647823 (2006).

[33] C.L. Kuo et al., Improved Measurements of the CMB Power Spectrum with ACBAR, arXiv: astro-ph/0611198 (2006).

[34] L. Page et al., Three-Year Wilkinson Microwave Anisotropy Probe (WMAP) Observations: Polarization Analysis, Astrophys. J. Suppl. 170, 335 (2007).

[35] D.J.C. MacKay, Information Theory, Inference, and Learning Algorithms, Cambridge University Press (2003).

(see also http:www.inference.phy.cam.ac.uk/mackay/itprnn/book.html).

[36] D.N. Spergel et al. Wilkinson Microwave Anisotropy Probe (WMAP) Three Year Results: Implications for Cosmology, Astrophys. J. Suppl. 170, 377 (2007).

[37] M. Liguori et al. Testing Primordial Non-Gaussianity in CMB Anisotropies, Phys. Rev. D73, 043505 (2006). 